\title{
MEAN CURVATURE FLOW OF MONOTONE LAGRANGIAN SUBMANIFOLDS
}

\author{
K. GROH, M. SCHWARZ, K. SMOCZYK, AND K. ZEHMISCH
}

\begin{abstract}
We use holomorphic disks to describe the formation of singularities in the mean curvature flow of monotone Lagrangian submanifolds in $\mathbb{C}^{n}$.
\end{abstract}

\section{INTRODUCTION}

Let $F_{0}: L \rightarrow \mathbb{C}^{n}$ be a smooth Lagrangian immersion of a compact, oriented manifold $L$ without boundary, i.e. $\operatorname{dim}(L)=n$ and $F_{0}^{*} \omega=0$, where $\omega(\cdot, \cdot)=$ $\langle J \cdot, \cdot\rangle$ is the standard symplectic form on $\mathbb{C}^{n}$ given by the composition of the complex structure $J$ and the euclidean metric $\langle\cdot, \cdot\rangle$.

Suppose $\left[0, T_{\text {sing }}\right)$ is the maximal time interval such that a smooth solution $F: L \times\left[0, T_{\text {sing }}\right) \rightarrow \mathbb{C}^{n}$ of the mean curvature flow equation

$$
\begin{gathered}
\frac{d}{d t} F=\vec{H} \\
F(\cdot, 0)=F_{0}
\end{gathered}
$$

exists. It is well known that $0<T_{\text {sing }}<\infty$ and that $L_{t}:=F_{t}(L)$ is Lagrangian, where we set $F_{t}:=F(\cdot, t)$. Moreover, we have

$$
\limsup _{t \rightarrow T_{\text {sing }}}|A|^{2}=\infty
$$

where $A=\nabla d F$ is the second fundamental form.

At the singular time $T_{\text {sing }}$ a singularity will form. The main aim of this paper is to understand the nature of singularities that monotone Lagrangian submanifolds might develop under various additional assumptions. This is motivated by a strong link between monotone Lagrangians and self-similar solutions of the Lagrangian mean curvature flow. To explain this relation, let us first recall the notion of blow-up points for the mean curvature flow and the notion of monotone Lagrangian submanifolds in symplectic geometry.

Date: June 18, 2006.

1991 Mathematics Subject Classification. Primary 53C44;

Supported by DFG, priority program SPP 1154, SM 78/1-1. 
To understand what happens as a singularity forms under the mean curvature flow in euclidean space, one introduces the notion of blow-up points. By definition, a blow-up point $p \in \mathbb{C}^{n}$ is a point such that one can find $x \in L$ with $F(x, t) \rightarrow p$ as $t \rightarrow T_{\text {sing }}$ and $|A|(x, t)$ becomes unbounded as $t \rightarrow T_{\text {sing. }}$. From the evolution equation of the second fundamental form one immediately deduces for $T_{\text {sing }}<\infty$ that

$$
\sup _{L_{t}}|A|^{2} \geq \frac{c}{T_{\text {sing }}-t}
$$

for a positive constant $c$ and all $0 \leq t<T_{\text {sing. Following Huisken [12, a }}$ singularity forms under the so-called type-1 condition, if

$$
\sup _{L_{t}}|A|^{2} \leq \frac{c}{T_{\text {sing }}-t}
$$

for some (different) constant $c>0$. All remaining cases of singularities with a different blow-up rate are called of type- 2 .

In 12, in the analogous context of mean curvature flow for hypersurfaces in $\mathbb{R}^{n+1}$, Huisken has given an answer to the question what happens if a singularity forms under the type- 1 hypothesis. This holds true as well in higher codimension, in particular also for Lagrangian submanifolds.

In this situation, a singularity forming in euclidean space looks like a selfsimilarly contracting solution (see definition below) after an appropriate rescaling procedure. To make this precise in our context, we assume for simplicity that the origin $O \in \mathbb{C}^{n}$ is a blow-up point for our flow of Lagrangian immersions (since the flow is invariant under isometries, we may assume w.l.o.g. that the blow-up point is given by the origin). Then, as in 12, we define the rescaled immersions $\widetilde{F}(x, s)$ by

$$
\begin{gathered}
\widetilde{F}(x, s):=\left(2\left(T_{\text {sing }}-t\right)\right)^{-1 / 2} F(x, t), \\
s(t):=-\frac{1}{2} \log \left(T_{\text {sing }}-t\right) .
\end{gathered}
$$

The submanifolds $\widetilde{L}_{s}:=\widetilde{F}(L, s)$ are defined for $-\frac{1}{2} \log T_{\text {sing }} \leq s<\infty$ and satisfy the equation

$$
\frac{d}{d s} \widetilde{F}(x, s)=\widetilde{\vec{H}}(x, s)+\widetilde{F}(x, s)
$$

where $\widetilde{\vec{H}}$ is the mean curvature vector of $\widetilde{L}_{s}$.

Under the type-1 assumption (1.1) one can prove as in [12] that for each sequence $s_{j} \rightarrow \infty$ there is a subsequence $s_{j_{k}}$ such that $\widetilde{L}_{s_{j_{k}}}$ converges to an immersed non-empty smooth limiting Lagrangian $\widetilde{L}_{\infty}$. In particular, this limit Lagrangian is a self-similarly contracting solution. A solution $F(x, t)$ of (MCF) is called self-similar, if we can find a rescaling $\widetilde{F}(x, t)=\psi(t) F(x, t)$, $\psi(t) \in \mathbb{R}$ such that all normal components of $\frac{d}{d t} \widetilde{F}$ vanish. This means that 
the images of $F$ evolve just by homotheties. Self-similar solutions of the mean curvature flow are determined by solutions of the following quasilinear elliptic system

$$
\vec{H}=-\frac{\varepsilon}{2} F^{\perp},
$$

with some constant $\varepsilon$. Under the flow, $\varepsilon$ will then vary with time but will keep its sign. In principle, one has to destinguish 3 cases. A solution is called self-contracting, trivial (i.e. minimal) or self-expanding according to whether $\varepsilon>0, \varepsilon=0$ or $\varepsilon<0$.

In the Lagrangian setting, we can reformulate equation (1.3) on $L$. A selfsimilar solution must satisfy the following relation between 1-forms on $L$

$$
H:=F^{*}(\omega(\cdot, \vec{H}))=-\frac{\varepsilon}{2} F^{*}(\omega(\cdot, F)) .
$$

$H$ is the mean curvature form. For the exact symplectic form $\omega=d \lambda$, $\lambda=\frac{1}{2}(x d y-y d x)$ on $\mathbb{C}^{n}$, it follows that

$$
H=\varepsilon F^{*} \lambda .
$$

This is due to the fact that rescaling is performed by the flow of the Liouville vector field $F$, i.e. $\mathcal{L}_{F} \omega=\omega$. Note also that the Lagrangian submanifolds under consideration may be noncompact.

The mean curvature form $H$ is closed. Moreover, by a result of Morvan [13. (see also [5] for a recent extension), the complex volume form $d z$ on $\mathbb{C}^{n}$ satisfies

$$
d z_{\mid L}=e^{i \alpha} d \mu, \quad d \alpha=H,
$$

where $\alpha$ is the locally defined Lagrangian angle of $L$ and $d \mu$ denotes the induced volume form on $L$.

In the Lagrangian context, there is a strong connection between self-similarly contracting solutions and monotone Lagrangian submanifolds. We recall the following definition (cf. [15]):

Definition 1.1. Assume $F: L \rightarrow \mathbb{C}^{n}$ is Lagrangian. Suppose $u: \mathbb{D} \rightarrow \mathbb{C}^{n}$ is a smooth map of the 2-disk $\mathbb{D}$ whose boundary $\partial u:=u(\partial \mathbb{D})$ is contained in $L_{0}:=F(L)$. Let $\mu(u)$ be the Maslov index of $u$ and $\omega(u):=\int_{\mathbb{D}} u^{*} \omega$ its symplectic area. $L_{0}$ is called monotone, if there exists a positive constant $\varepsilon>0$ (called monotonicity constant) such that

$$
\mu(u)=\frac{\varepsilon}{\pi} \omega(u)
$$

on all such disks $u$.

Remark 1.2. Assume $u$ is a disk with boundary curve $\gamma \subset L$ and that $F: L \rightarrow\left(\mathbb{C}^{n}, \omega=d \lambda\right)$ is Lagrangian. By Stokes' theorem the symplectic area is

$$
\omega(u)=\int_{\gamma} F^{*} \lambda
$$


and by Morvan's result [13] we have $H(\gamma)=\pi \mu(u)$ so that (1.5) becomes

$$
[H]=\varepsilon\left[F^{*} \lambda\right] .
$$

In particular, by (1.6), self-similarly contracting solutions are monotone. Moreover, if $L_{0}$ is monotone with monotonicity constant $\varepsilon$, then for $\tilde{L}_{0}:=$ $c L_{0}$ we have $\tilde{H}=H, \tilde{F}^{*} \lambda=c^{2} F^{*} \lambda$, so that $\tilde{L}_{0}$ is monotone with monotonicity constant $\tilde{\varepsilon}=c^{-2} \varepsilon$. Thus, by a simple rescaling argument we can always assume that the monotonicity constant $\varepsilon$ of a monotone Lagrangian submanifold satisfies $\varepsilon=1$.

Remark 1.3. If $\gamma_{1}, \gamma_{2}$ are two closed curves on $L$ and $\gamma_{1}$ is homotopic to $\gamma_{2}$, then $H\left(\gamma_{1}\right)=H\left(\gamma_{2}\right)$ and by Stokes' theorem and the Lagrangian property of $L$ also $\lambda_{\mid L}\left(\gamma_{1}\right)=\lambda_{\mid L}\left(\gamma_{2}\right)$. In particular, if $u: \mathbb{D} \rightarrow \mathbb{C}^{n}, \partial u \subset L$ is a holomorphic disk with boundary on $L$, then $\lambda_{\mid L}(\partial u)=\omega(u)>0$ and $\lambda_{\mid L}(\gamma)>0$ for any curve $\gamma$ homotopic to $\partial u$.

Since self-similarly contracting solutions are monotone and self-similar solutions appear as type-1 blow-ups, it is natural to ask the following:

Does the flow preserve the monotonicity of Lagrangian submanifolds and will monotone Lagrangians develop type-1 singularities?

We will see, as stated in the next proposition, that the first part of this question has a positive answer whereas the second part is wrong in general.

Proposition 1.4. Suppose $F: L \times\left[0, T_{\text {sing }}\right) \rightarrow \mathbb{C}^{n}$ is a solution of the Lagrangian mean curvature flow. If $L_{0}=F(L, 0)$ is monotone with monotonicity constant $\varepsilon_{0}$, then $\left[F^{*} \lambda\right]=\frac{1}{\varepsilon_{0}}\left(1-\varepsilon_{0} t\right)[H]$ for any $t \in\left[0, T_{\text {sing }}\right)$. In particular, $L_{t}=F(L, t)$ remains monotone for $t \in\left[0, \frac{1}{\varepsilon_{0}}\right)$, but with the monotonicity constant $\varepsilon_{t}:=\frac{\varepsilon_{0}}{1-\varepsilon_{0} t}>0$.

From this proposition and Remark 1.3 we conclude that the areas of holomorphic disks with boundary on a monotone Lagrangian torus (if they exist) all shrink with the same rate. This means that they would all disappear at the same time $t=\frac{1}{\varepsilon}$. Therefore, one is tempted to conjecture that monotone tori will completely shrink to a single point and will always develop a type1 singularity at $T_{\operatorname{sing}}=\frac{1}{\varepsilon}$, which after blow-up looks like a self-similarly shrinking Lagrangian torus. That this is not the case, even under strong additional geometric assumptions, is somehow surprising. Even under the assumption that the Lagrangian submanifolds admit quasi-fillings (see definition below) for some homology class $e \in H_{1}(L ; \mathbb{Z})$ one still might encounter type-2 singularities. This will be demonstrated for examples of embedded, equivariant, monotone 2-tori that we discuss in this article.

Since the existence of holomorphic disks with boundary on the Lagrangian submanifold is important, the next theorem due to Gromov [11] for embedded Lagrangian submanifolds is of particular interest. 
Theorem 1.5. (Gromov) Suppose $L \subset \mathbb{C}^{n}$ is an embedded closed Lagrangian. Then there exists at least one holomorphic disk $u: \mathbb{D} \rightarrow \mathbb{C}^{n}$ whose boundary is contained in $L$. In particular, $\left[\lambda_{\mid L}\right] \neq 0$.

In view of Gromov's theorem and of Remark 1.3 one would like to control the embeddedness of Lagrangian submanifolds under the mean curvature flow. It is well-known that embeddedness is preserved for hypersurfaces moving by their mean curvature and that this, in general, is no longer true in higher codimensions. Therefore we define a constant $T_{\mathrm{emb}}$ as follows:

Suppose $F_{0}: L \rightarrow \mathbb{C}^{n}$ is a Lagrangian embedding and that $F: L \times$ $\left[0, T_{\text {sing }}\right) \rightarrow \mathbb{C}^{n}$ is the maximal smooth solution of the mean curvature flow. Then we set

$$
T_{\mathrm{emb}}:=\sup \left\{t \in\left[0, T_{\text {sing }}\right): L_{\tau} \text { is embedded for } \tau \in[0, t]\right\} .
$$

By definition one has

$$
0<T_{\mathrm{emb}} \leq T_{\text {sing }}
$$

It turns out that embeddedness is important in the context of monotone Lagrangian submanifolds. We will prove the following result:

Theorem 1.6. Suppose $F_{0}: L \rightarrow \mathbb{C}^{n}$ is an embedding of a compact, oriented, monotone Lagrangian submanifold with monotonicity constant $\varepsilon>0$. Then $T_{e m b} \leq \frac{1}{\varepsilon}$.

The proof of this theorem will be carried out in Section 2 and is based on Gromov's result mentioned above.

A corollary of Theorem [1.6 is:

Corollary 1.7. Suppose $F_{0}: L \rightarrow \mathbb{C}^{n}$ is an embedding of a compact, oriented, monotone Lagrangian submanifold with monotonicity constant $\varepsilon>0$. If $T_{\text {emb }}=T_{\text {sing }}$, i.e. if the solution stays embedded for all $t<T_{\text {sing }}$, then $T_{\text {sing }} \leq \frac{1}{\varepsilon}$.

An obvious question is, if $T_{\text {sing }}>\frac{1}{\varepsilon}$ is possible at all, or if for any monotone Lagrangian we must have $T_{\text {sing }} \leq \frac{1}{\varepsilon}$ ? The figure eight curve depicted in Figure 1 shows that, at least in the immersed case, $\varepsilon$ need not be related to $T_{\text {sing. }}$

Another obvious question arising from Corollary 1.7 is, if - under the same assumptions as in Corollary 1.7 - we must have $T_{\text {sing }}=\frac{1}{\varepsilon}$ or if $T_{\text {sing }}<\frac{1}{\varepsilon}$ is possible. In this article we will give examples with $T_{\mathrm{emb}}=T_{\mathrm{sing}}<\frac{1}{\varepsilon}$ that develop type- 2 singularities.

On the other hand, it is possible to characterize the solutions with $T_{\text {sing }}=\frac{1}{\varepsilon}$ by the following proposition (for a proof see Section 2): 


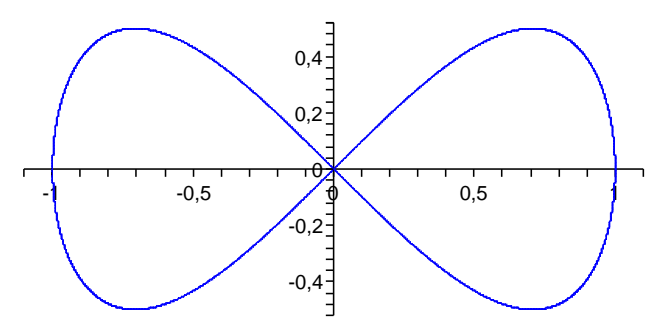

Figure 1. For the figure eight curve $\gamma$ we have $\left[\lambda_{\mid \gamma}\right]=[H]=$ 0 , so that $[H]=\varepsilon\left[\lambda_{\mid L}\right]$ holds for any $\varepsilon \in \mathbb{R}$. Consequently, $\gamma$ is monotone and the monotonicity constant is not related to the singular time $T_{\text {sing. }}$.

Proposition 1.8. Suppose $F_{0}: L \rightarrow \mathbb{C}^{n}$ is an immersion of a compact, oriented, monotone Lagrangian submanifold with monotonicity constant $\varepsilon>$ 0 . Then $T_{\text {sing }}=\frac{1}{\varepsilon}$, if and only if the rescaled flow (1.2) is Hamiltonian.

In 14] Neves proved that a Lagrangian submanifold with trivial Maslov class does not develop a type- 1 singularity in finite time. His proof is based on methods from geometric measure theory. We will give a very elementary proof for this, in particular we will prove the following extension of Neves' result:

Theorem 1.9. Let $F: L \rightarrow \mathbb{C}^{n}$ be a Lagrangian self-similar solution, i.e. a solution of

$$
\vec{H}=-\frac{\varepsilon}{2} F^{\perp}
$$

with $\varepsilon \neq 0^{1)}$. Suppose $L$ has trivial Maslov class $\mu=\frac{1}{\pi}[H]=\frac{1}{\pi}[d \alpha]=0$ and that there exist constants $c>0, \sigma<\varepsilon$ such that the Lagrangian angle $\alpha$ satisfies $\left|\nabla^{k} \alpha\right|^{2} \leq c e^{\frac{\sigma|F|^{2}}{4}}$ for $0 \leq k \leq 2$. Then $L$ is part of a minimal cone.

The proof of this theorem is very short and merely uses partial integration w.r.t. the Gauß kernel. Note, that the assumption on $\left|\nabla^{k} \alpha\right|^{2}$ is automatically satisfied for a type- 1 blow-up. Note also that it is an open problem in the closed case to determine which Lagrangians admit $\mu=0$.

For the formulation of the next theorem we need the following definition.

\footnotetext{
1) $\varepsilon$ can be either positive (contracting solution) or negative (expanding solution)
} 
Definition 1.10. A type-1 singularity will be called embedded, if $T_{\mathrm{emb}}=$ $T_{\text {sing }}$ and if in addition, one of the type-1 blow-up limiting Lagrangians obtained by the rescaled flow (1.2) is embedded as well.

We will prove:

Theorem 1.11. Let $F_{0}: L \rightarrow \mathbb{C}^{n}$ be an embedding of a compact, oriented, monotone Lagrangian submanifold with monotonicity constant $\varepsilon$. Suppose the Lagrangian submanifold develops an embedded type-1 singularity. Then $T_{\text {sing }}=\frac{1}{\varepsilon}$.

However, even under the assumptions made in Theorem 1.11 it is still unclear, if the singular set consists of a single point and if the blow-up converges to a compact self-similar Lagrangian. This very much depends on the "families" of holomorphic disks that can be attached to the evolving submanifolds. For example, in the easiest case of a Lagrangian $L \subset \mathbb{C}^{2}$ it is in general unclear, if for any homology class $e \in H_{1}(L ; \mathbb{Z})$ there exists an embedding

$$
f:\left(S^{1} \times \mathbb{D}, S^{1} \times \partial \mathbb{D}\right) \longrightarrow\left(\mathbb{C}^{2}, L\right),
$$

such that the maps $f_{\tau}=f(\tau,$.$) are holomorphic in the usual sense for all$ $\tau \in S^{1}$ and $\left[\partial f_{\tau}\right]=e$. Such a map (or rather its image) is called an $e$-filling of $L$.

The standard examples of fillings in $\mathbb{C}^{2}$ are for the Clifford torus $T_{\pi / 2}=$ $\left\{\left|z_{1}\right|^{2}+\left|z_{2}\right|^{2}=1\right\}$, namely

$$
f_{1}(\tau, z)=\frac{1}{\sqrt{2}}\left(z, e^{i \tau}\right) \quad \text { and } \quad f_{2}(\tau, z)=\frac{1}{\sqrt{2}}\left(e^{i \tau}, z\right) .
$$

Notice that the Maslov index for both cycles is

$$
\mu_{T_{\pi / 2}}\left(\left[\partial f_{j}\right]\right)=2, j=1,2 .
$$

In general it is unknown, if such fillings survive under Hamiltonian deformations. If one is only interested in the existence of sufficiently many holomorphic disks attached to Lagrangian submanifolds such that the boundary maps represent certain homology classes, the following relative Gromov invariant type notion is useful.

We will say that $L \subset \mathbb{C}^{2}$ admits an e-quasi-filling for a homology class $e \in H_{1}(L ; \mathbb{Z})$, if for all points $p \in L$ there exists a holomorphic disk

$$
u_{p}:(\mathbb{D}, \partial \mathbb{D}, 1) \longrightarrow\left(\mathbb{C}^{2}, L, p\right)
$$

such that $\left[\partial u_{p}\right]=e$. For example the following disk families

$$
f_{a}(\tau, z)=\frac{1}{\sqrt{2}}\left(z, e^{i \tau} \frac{z-a}{1-\bar{a} z}\right) \quad \text { for } a \in \mathbb{C},|a|<1
$$


which satisfy

$$
\mu_{T_{\pi / 2}}\left(\left[\partial f_{a}\right]\right)=4
$$

define quasi-fillings for the Clifford torus with respect to the product class of the defining circles. As all disks $f_{a}(\tau, \mathbb{D})$ intersect in the singularity $(0, a)$ this is not a filling.

We remark that up to parameterizations, the disk families in (1.7) and (1.8) constitute a complete list of all unparameterized holomorphic disks attached to the Clifford torus.

It seems that the existence of quasi-fillings depends on the Hamiltonian isotopy class as the following statement due to Gromov [1] (cf. 8, Proposition 4.1.A] and [22]) suggests.

Theorem 1.12 (Gromov). Let $L$ be an embedded Lagrangian torus in $\mathbb{C}^{2}$ Hamiltonian isotopic to the Clifford torus $T_{\pi / 2}$ via $\varphi$. Then for $j=1,2$ the monotone torus $L$ admits a $\varphi_{*} e_{j}$-quasi-filling, where $e_{1}, e_{2}$ are the generators of $H_{1}\left(T_{\pi / 2} ; \mathbb{Z}\right)$ corresponding to the defining circles.

Hence, any Lagrangian torus Hamiltonian isotopic to the Clifford torus carries two different holomorphic disk families with Maslov index 2.

This theorem can be used to show that not all embedded Lagrangian tori are symplectomorphic. We will now give additional examples of monotone tori. Let $z: S^{1} \rightarrow \mathbb{C}^{*}$ be a closed curve. Using complex-symplectic coordinates on $\mathbb{C}^{2}$ we obtain a Lagrangian torus through the map $F: \mathbb{T}^{2} \rightarrow \mathbb{C}^{2}$,

$$
F(\phi, \psi):=(z(\phi) \cos \psi, z(\phi) \sin \psi) .
$$

The embedded Lagrangian torus generated by the curve $z(\phi)=e^{i \phi}$ is symplectomorphic to the Clifford torus $T_{\pi / 2}=\left\{\left|z_{1}\right|^{2}+\left|z_{2}\right|^{2}=1\right\}$ by the $\mathrm{SU}_{2^{-}}$ transformation matrix

$$
S=\frac{1}{\sqrt{2}}\left(\begin{array}{cc}
1 & i \\
i & 1
\end{array}\right) .
$$

A second example is given by what we call the Chekanov torus $T_{1}^{2}(\pi / 2)$, see [3. In this case consider the curve in the open right half plane

$$
z(\phi)=e^{i \kappa \cos (\phi)} \cos (\phi)+i \kappa e^{-i \kappa \cos (\phi)} \sin (\phi), \quad \text { where } \kappa=\frac{1}{\sqrt{2}} .
$$

This curve $z$ is embedded with $\{z\} \cap\{-z\}=\emptyset$. The Chekanov torus $T_{1}^{2}(\pi / 2)$ is the embedded equivariant torus generated by $z$, see 22]. Equivalently, we mention the Eliashberg-Polterovich torus $L_{\Gamma}^{0}$ considered in [8], which is symplectically isomorphic to the Chekanov torus. Denote by $\Gamma$ the boundary curve of an embedded disk $D$ in $\mathbb{C}$ such that $\bar{D}$ does not contain the origin. By definition $L_{\Gamma}^{0}$ is the intersection of the sub-level set of $\Gamma$ under the function $\left(z_{1}, z_{2}\right) \mapsto z_{1} z_{2}$ with the sub-level set of zero under the Hamiltonian 
function $H\left(z_{1}, z_{2}\right)=\left|z_{1}\right|^{2}-\left|z_{2}\right|^{2}$. To see the equivariant picture of this, let $w: S^{1} \longrightarrow \mathbb{C}^{*}$ be a regular parameterisation of $\Gamma$ and choose a branch of square-root defined near $D$. Then

$$
L_{\Gamma}^{0}=\left\{\left(\sqrt{w(\phi)} e^{i t}, \sqrt{w(\phi)} e^{-i t}\right)\right\}_{\phi, t \in S^{1}} .
$$

Under the $\mathrm{U}_{2}$-transformation matrix

$$
U=\frac{1}{\sqrt{2}}\left(\begin{array}{cc}
1 & i \\
1 & -i
\end{array}\right)=\left(\begin{array}{cc}
1 & 0 \\
0 & -i
\end{array}\right) S
$$

the torus $E Q_{z^{\text {int }}}$ corresponding to the curve $z^{\text {int }}=\sqrt{2 w}$ is mapped to $L_{\Gamma}^{0}$. Hence, the torus $E Q_{z^{\text {int }}}$ is symplectomorphic to $L_{\Gamma}^{0}$.

The reason why Chekanov and Eliashberg-Polterovich considered these tori is that they were the first examples of monotone Lagrangian tori not symplectomorphic to the Clifford torus. Both tori, $T_{1}^{2}(\pi / 2)$ and $L_{\Gamma}^{0}$, (which are symplectomorphic) are so-called exotic tori. The exoticness follows from Theorem 1.12 because $L_{\Gamma}^{0}$ allows only one holomorphic disk family with Maslov index 2. Further, for the tori $L_{\Gamma}^{0}$ we have generating cycles

$$
c(\phi)=(\sqrt{w(\phi)}, \sqrt{w(\phi)})
$$

for $\phi \in S^{1}$. In contrast to Theorem 1.12 we have the following statement, which can be obtained via similar methods (see [22]):

Theorem 1.13. Let $L$ be an embedded Lagrangian torus in $\mathbb{C}^{2}$ symplectomorphic to the exotic torus $L_{\Gamma}^{0}$ via $\varphi$. Then the monotone torus $L$ admits a $\varphi_{*} e$-quasi-filling, where the homology class $e \in H_{1}\left(L_{\Gamma}^{0} ; \mathbb{Z}\right)$ is represented by (1.9).

An important question is if the existence of $e_{i}$-quasi-fillings of an embedded monotone Lagrangian torus $L \subset \mathbb{C}^{n}$ for a full set of generators $e_{1}, \ldots, e_{n} \in$ $H_{1}(L ; \mathbb{Z})$ can be used to detect the type of singularity that it will develop under the mean curvature flow. We will see that this is not the case (note that the basis of generators is in general not canonical).

Essentially, our article consists of two parts. In the first part we will derive some results for the Lagrangian mean curvature flow in general, whereas in the second part we restrict our attention to an equivariant situation to find examples of type- 1 and type- 2 singularities of monotone Lagrangian submanifolds $L \subset \mathbb{C}^{n}$ and in particular of embedded, monotone Lagrangian tori $\mathbb{T}^{2} \subset \mathbb{C}^{2}$. The equivariant situation is a generalization of the one discussed above and can be described as follows.

Let $L:=S^{1} \times S^{n-1}$ and assume that 


$$
\begin{array}{r}
z_{0}: S^{1} \rightarrow \mathbb{C}^{*} \\
z_{0}(\phi):=u_{0}(\phi)+i v_{0}(\phi)
\end{array}
$$

is some closed immersed curve in $\mathbb{C}^{*}=\mathbb{C} \backslash\{0\}$. The equivariant Lagrangian immersions $L_{0}=F_{0}\left(S^{1} \times S^{n-1}\right)$ that we study in the second part of this article, are of the following form. Define an immersion $F_{0}: S^{1} \times S^{n-1} \rightarrow \mathbb{C}^{n}$ by

$$
F_{0}(\phi, x):=\left(u_{0}(\phi) G(x), v_{0}(\phi) G(x)\right),
$$

where we assume that the complex structure $J$ is acting on $\mathbb{C}^{n}$ by

$$
J\left(x^{1}, \ldots, x^{n}, y^{1}, \ldots, y^{n}\right):=\left(-y^{1}, \ldots,-y^{n}, x^{1}, \ldots, x^{n}\right)
$$

and that $G: S^{n-1} \rightarrow \mathbb{R}^{n}$ is the standard embedding of the sphere of radius 1 in $\mathbb{R}^{n}$. In particular, in dimension $n=2$ we get nice examples of Lagrangian tori in $\mathbb{C}^{2}$.

Since the mean curvature flow is isotropic, the condition to be equivariant is preserved under the flow. This implies that in this situation the flow will be determined by the flow of the corresponding profile curves $\gamma_{t}:=z_{t}\left(S^{1}\right)$, $z_{t}:=u_{t}+i v_{t}: S^{1} \rightarrow \mathbb{C}^{*}$ induced by

$$
F_{t}(\phi, x)=\left(u_{t}(\phi) G(x), v_{t}(\phi) G(x)\right) .
$$

As will be shown in Section 3.1, the equivariant Lagrangian mean curvature flow can be reduced to the following flow of the profile curves in $\mathbb{C}^{*}$

$$
\begin{gathered}
\frac{d}{d t} z=-f \nu, \\
z(\cdot, 0)=z_{0}(\cdot), \\
\text { with } f:=k+(n-1) \frac{\langle z, \nu\rangle}{|z|^{2}} .
\end{gathered}
$$

Here, $\nu$ denotes the outward pointing unit normal along the curve $\gamma_{t}:=$ $z_{t}\left(S^{1}\right), z_{t}(\phi)=z(\phi, t)$ and $k$ is the curvature of $\gamma_{t}$.

The reason to consider equivariant solutions is to get simple, non-trivial examples of monotone solutions that develop type- 1 or type- 2 singularities. There are essentially two classes of closed embedded profile curves, those who contain the origin in their interior and those who do not. In dimension $n=2$ the Clifford torus belongs to the first class and the exotic tori to the 
second class. In particular, these classes are not symplectomorphic to each other.

For the first class of profile curves we will show that neither starshapedness, embeddedness, monotonicity or a natural convexity assumption are sufficient conditions for the contraction to a single point (which would have to be the origin in view of the point-symmetry). In particular, even under geometrically very restrictive conditions we will encounter type-2 singularities. For the second class of curves the first author proves in his $\mathrm{PhD}$ thesis [10] that they contract in finite time to a single point $p \in \mathbb{C}^{*}$, if $f>0$.

On the other hand we can prove fairly general regularity theorems. For example, one of our main results is:

Theorem 1.14. Let $F: S^{1} \times S^{n-1} \times\left[0, T_{\text {sing }}\right) \rightarrow \mathbb{C}^{n}, n \geq 2$ be a smooth solution of the equivariant Lagrangian mean curvature flow with corresponding profile curves $z: S^{1} \rightarrow \mathbb{C}^{*}$. If the initial profile curve is starshaped ${ }^{2)}$ w.r.t. the origin, then

$$
\liminf _{t \rightarrow T_{\text {sing }}} r=0,
$$

where $\left[0, T_{\text {sing }}\right)$ is the maximal time interval on which a smooth solution exists and $r:=|z|$.

This theorem states that for $n \geq 2$ the first singularity of a starshaped curve must occur at the origin.

Is is interesting to ask, if there exists a class of monotone Lagrangian submanifolds that develop type-1 singularities. Let us define

$$
\begin{aligned}
\mathscr{E}:=\left\{L \subset \mathbb{C}^{n}:\right. & L \text { is a monotone, equivariant Lagrangian } \\
& \text { immersion with }|\vec{H}|>0 \text { and such that the } \\
& \text { profile curve } \left.z: S^{1} \rightarrow \mathbb{C}^{*} \text { is starshaped }\right\}
\end{aligned}
$$

Obviously, a Lagrangian $L \subset \mathscr{E}$ satisfies very strong geometric conditions. Therefore, it is surprising that even within this class both types of singularities occur; to be precise we will prove the following theorem:

\section{Theorem 1.15.}

(i) The class $\mathscr{E}$ is stable under the mean curvature flow and there exist embedded $L_{1}, L_{2} \subset \mathscr{E}$ such that $L_{1}$ develops a singularity of type-1 and $L_{2}$ a singularity of type-2 at the origin of $\mathbb{C}^{n}$.

\footnotetext{
${ }^{2)}$ In this paper, a curve $\gamma=z\left(S^{1}\right)$ will be called starshaped, if $\langle z, \nu\rangle>0$ everywhere on $\gamma$, no matter if $\gamma$ is embedded or immersed.
} 
(ii) Let $z: S^{1} \rightarrow \mathbb{C}^{*}$ be the initial profile curve of a Lagrangian $L \subset \mathscr{E}$ and let $l \geq 1+4 n \omega_{0}$ be a positive integer, where $\omega_{0}=$ wind $_{0}(z)$ is the winding number of $z$ w.r.t. the origin. In addition, suppose that $z$ is rotationally symmetric w.r.t. rotations by $\frac{2 \pi}{l}$, i.e. $z(\phi)=z\left(\phi+\frac{2 \pi}{l}\right)$, for all $\phi \in[0,2 \pi)$. Then the corresponding equivariant Lagrangian immersion $L \subset \mathscr{E}$ develops a type-1 singularity at the origin and the Lagrangian submanifold converges to the origin as $t \rightarrow T_{\text {sing }}=\frac{1}{\varepsilon}$, where $\varepsilon$ is the monotonicity constant of L. After rescaling, the profile curves converge smoothly to a smooth limiting curve $\tilde{\gamma}_{\infty}$ which is one of the self-similarly contracting solutions classified by Anciaux in [1].

The organization of our article is as follows. In Section 2 we will derive general results for the Lagrangian mean curvature flow. There we give proofs of Theorems and Propositions 1.4, 1.6, 1.8, 1.9] and 1.11.

In Section 3 we discuss the equivariant situation and prove that a number of geometric conditions are preserved under the equivariant flow. Finally, in the last section we give the proofs for Theorems 1.14 and 1.15

\section{MeAn CURVATURe Flow AND MONOTONICITy OF LAGRANGIAN SUBMANIFOLDS}

As in the introduction, let $F: L \times\left[0, T_{\text {sing }}\right) \rightarrow \mathbb{C}^{n}$ be a maximal smooth solution of

$$
\begin{gathered}
\frac{d}{d t} F=\vec{H} \\
F(\cdot, 0)=F_{0}
\end{gathered}
$$

for some compact, smooth manifold $L$. Moreover, for a Lagrangian immersion $F: L \rightarrow \mathbb{C}^{n}$ we set

$$
\begin{gathered}
F_{\alpha}:=\frac{\partial F}{\partial y^{\alpha}}, \\
F_{\alpha \beta}=\frac{\partial^{2} F}{\partial y^{\alpha} \partial y^{\beta}}
\end{gathered}
$$

and

$$
\nu_{\alpha}:=J F_{\alpha},
$$

where $\left(y^{\alpha}\right)_{\alpha=0, \ldots, n-1}$ are local coordinates for $L$. Then, by the Lagrangian condition, the vectors $\nu_{\alpha}$ are normal along $F(L)$. The induced metric $g_{\alpha \beta}$ and the second fundamental tensor $h_{\alpha \beta \gamma}$ on $L$ are given by $g_{\alpha \beta}=\left\langle F_{\alpha}, F_{\beta}\right\rangle$ resp. $h_{\alpha \beta \gamma}=\left\langle\nu_{\alpha}, F_{\beta \gamma}\right\rangle$. In local coordinates, the components $H_{\alpha}$ of the mean curvature form are given by $H_{\alpha}=g^{\beta \gamma} h_{\alpha \beta \gamma}$, where $g^{\beta \gamma}$ denotes the inverse of $g_{\beta \gamma}$. The mean curvature vector can be expressed as $\vec{H}=g^{\beta \gamma} H_{\beta} \nu_{\gamma}$. The following Lemma is well-known (cf. [17]). 
Lemma 2.1. Under the mean curvature flow in euclidean space, the mean curvature form evolves according to the evolution equation

$$
\frac{d}{d t} H=d d^{\dagger} H
$$

where $d^{\dagger}$ denotes the negative adjoint of $d$ w.r.t. the induced metric on $L_{t}$.

In addition, as we will now prove, the monotonicity is preserved, if one allows the monotonicity constant to vary with time. This was already stated in Proposition 1.4

Proof of Proposition 1.4. We need the evolution equation for $F^{*} \lambda$. One easily observes

$$
\left(F^{*} \lambda\right)_{\alpha}=\frac{1}{2}\left\langle J F, F_{\alpha}\right\rangle
$$

Then

$$
\begin{aligned}
2 \frac{d}{d t}\left(F^{*} \lambda\right)_{\alpha} & =\left\langle J \vec{H}, F_{\alpha}\right\rangle+\left\langle J F, \nabla_{\alpha}\left(H^{\gamma} \nu_{\gamma}\right)\right\rangle \\
& =-H_{\alpha}+\nabla_{\alpha} H^{\gamma}\left\langle J F, \nu_{\gamma}\right\rangle-H^{\gamma} h_{\alpha \gamma}^{\beta}\left\langle J F, F_{\beta}\right\rangle .
\end{aligned}
$$

To proceed, we need an expression for $d d^{\dagger}\left(F^{*} \lambda\right)=d\left(\nabla^{\alpha}\left(F^{*} \lambda\right)_{\alpha}\right)$. We compute

$$
2 \nabla_{\alpha}\left(F^{*} \lambda\right)_{\beta}=\left\langle\nu_{\alpha}, F_{\beta}\right\rangle+h_{\alpha \beta}^{\gamma}\left\langle J F, \nu_{\gamma}\right\rangle=h_{\alpha \beta}^{\gamma}\left\langle J F, \nu_{\gamma}\right\rangle
$$

and

$$
2 d^{\dagger}\left(F^{*} \lambda\right)=H^{\gamma}\left\langle J F, \nu_{\gamma}\right\rangle .
$$

Taking the exterior derivative yields

$$
2\left(d d^{\dagger}\left(F^{*} \lambda\right)\right)_{\alpha}=\nabla_{\alpha} H^{\gamma}\left\langle J F, \nu_{\gamma}\right\rangle+H_{\alpha}-H^{\gamma} h_{\alpha \gamma}^{\beta}\left\langle J F, F_{\beta}\right\rangle .
$$

Combining this with the above expression for $2 \frac{d}{d t}\left(F^{*} \lambda\right)_{\alpha}$ we obtain

$$
\frac{d}{d t} F^{*} \lambda=d d^{\dagger}\left(F^{*} \lambda\right)-H
$$

This and the evolution equation (2.1) imply

$$
\frac{d}{d t}\left(F^{*} \lambda-\frac{1}{\varepsilon_{0}}\left(1-\varepsilon_{0} t\right) H\right)=d d^{\dagger}\left(F^{*} \lambda-\frac{1}{\varepsilon_{0}}\left(1-\varepsilon_{0} t\right) H\right)
$$


By the monotonicity at $t=0$ we must have $\left[F^{*} \lambda\right]=\frac{1}{\varepsilon_{0}}[H]$ at $t=0$. The evolution equation above guarantees that the cohomology class of $F^{*} \lambda-$ $\frac{1}{\varepsilon_{0}}\left(1-\varepsilon_{0} t\right) H$ does not change. This proves

$$
\left[F^{*} \lambda\right]=\frac{1}{\varepsilon_{0}}\left(1-\varepsilon_{0} t\right)[H]
$$

for all $t \in\left[0, T_{\text {sing }}\right)$. Now as long as in addition $t \in\left[0, \frac{1}{\varepsilon_{0}}\right)$ we obtain

$$
[H]=\frac{\varepsilon_{0}}{1-\varepsilon_{0} t}\left[F^{*} \lambda\right]
$$

with

$$
\frac{\varepsilon_{0}}{1-\varepsilon_{0} t}>0
$$

which proves the lemma.

We are now ready to prove Theorem 1.6 .

Proof of Theorem [1.6. Suppose $T_{\mathrm{emb}}>\frac{1}{\varepsilon}$. Then $T_{\operatorname{sing}}>\frac{1}{\varepsilon}$ and $L_{\frac{1}{\varepsilon}}$ is embedded. From Proposition 1.4 we conclude $\left[F^{*} \lambda\right]=0$ at $t=\frac{1}{\varepsilon}$. By Theorem 1.5 $L_{\frac{1}{\varepsilon}}$ cannot be embedded. This contradiction proves $T_{\mathrm{emb}} \leq \frac{1}{\varepsilon}$.

Proof of Proposition 1.8. Suppose $F: L \times\left[0, T_{\text {sing }}\right) \rightarrow \mathbb{C}^{n}$ is a solution of the Lagrangian mean curvature flow and that $L_{0}=F(L, 0)$ is monotone with monotonicity constant $\varepsilon$. By Proposition 1.4 we then have $\left[F^{*} \lambda\right]=\frac{1-\varepsilon t}{\varepsilon}[H]$ for any $t \in\left[0, T_{\text {sing }}\right)$. For the rescaled flow $\widetilde{F}=\left(2\left(T_{\text {sing }}-t\right)\right)^{-\frac{1}{2}}$ we get

$$
F^{*} \lambda=2\left(T_{\text {sing }}-t\right) \widetilde{F}^{*} \lambda, \quad H=\widetilde{H},
$$

so that

$$
\left[\widetilde{F}^{*} \lambda\right]=\frac{1-\varepsilon t}{2 \varepsilon\left(T_{\text {sing }}-t\right)}[\widetilde{H}]
$$

On the other hand, it is well known that a variation of Lagrangian immersions $\widetilde{F}_{s}: L \rightarrow \mathbb{C}^{n}$ is Hamiltonian, if and only if

$$
\left[\widetilde{F}^{*}\left(\omega\left(\cdot, \frac{d}{d s} \widetilde{F}\right)\right)\right]=0
$$

For the rescaled flow we have

$$
\left[\widetilde{F}^{*}\left(\omega\left(\cdot, \frac{d}{d s} \widetilde{F}\right)\right)\right]=\left[\widetilde{H}-2 \widetilde{F}^{*} \lambda\right]
$$

and in view of (2.4) this vanishes, if and only if $T_{\text {sing }}=\frac{1}{\varepsilon}$.

Proof of Theorem 1.9. By assumption the mean curvature form satisfies

$$
H=d \alpha
$$


with

$$
H=\varepsilon F^{*} \lambda
$$

This implies

$$
d^{\dagger} H=\Delta \alpha=\varepsilon d^{\dagger} F^{*} \lambda=\varepsilon\langle d \alpha, d s\rangle
$$

with $s:=\frac{|F|^{2}}{4}$. We multiply this equation with $\alpha e^{-\varepsilon s}$ and integrate on $L$. Note, that the integral exists in view of the curvature estimate for $\left|\nabla^{k} \alpha\right|^{2}$. This gives

$$
\int_{L} \alpha \Delta \alpha e^{-\varepsilon s} d \mu=\int_{L} \varepsilon \alpha\langle d \alpha, d s\rangle e^{-\varepsilon s} d \mu .
$$

By assumption $\left|\nabla^{k} \alpha\right|^{2} \leq c e^{\sigma s}$ with $\sigma<\varepsilon$ so that we may integrate by parts in the first integral without getting boundary terms at infinity. This yields

$$
\int_{L}|H|^{2} e^{-\varepsilon s} d \mu=0
$$

hence $H=\varepsilon F^{*} \lambda=0$ which means that $L$ is part of a minimal cone.

\section{Proof of Theorem 1.11,}

Suppose $L_{t}$ develops an embedded type- 1 singularity at the origin and that $\tilde{L}_{\infty}$ is an embedded limiting Lagrangian of the rescaled flow $\tilde{L}_{s}, s \in\left[s_{0}, \infty\right)$ with $s_{0}:=-\frac{1}{2} \log T_{\text {sing. }}$. Then by Huisken's result we know $\tilde{H}_{\infty}=2 \tilde{\lambda}_{\infty}$.

If $\left[\widetilde{H}_{\infty}\right]=0$, then in view of Theorem $1.9 \tilde{L}_{\infty}$ would be part of a minimal cone. On the other hand it is well-known that a type-1 blow-up of a compact submanifold is smooth, complete and not totally geodesic but any smooth, complete part of a minimal cone is totally geodesic. So we must have $\left[\widetilde{H}_{\infty}\right] \neq$ 0 .

$\left[\widetilde{H}_{\infty}\right] \neq 0$ and $\widetilde{H}_{\infty}=2 \widetilde{\lambda}_{\infty}$ implies that there exists a closed embedded curve $\widetilde{\gamma} \subset \widetilde{L}_{\infty}$ such that $\int_{\widetilde{\gamma}} \tilde{\lambda}_{\infty} \neq 0$. Choose a ball $B(0, R) \subset \mathbb{C}^{n}$ with $\widetilde{\gamma} \subset B(0, R)$ for some $R>0$. Since $\widetilde{L}_{\infty}$ is an embedded limiting Lagrangian submanifold, there exist homology classes $\widetilde{e}_{\infty} \in H_{1}\left(\widetilde{L}_{\infty} ; \mathbb{Z}\right), e \subset H_{1}(L ; \mathbb{Z})$, a closed embedded curve $\widetilde{\gamma}_{\infty} \in \widetilde{e}_{\infty}$ and a sequence of closed embedded curves $\gamma_{t} \in e$ such that $\widetilde{\gamma}_{\infty} \subset B(0, R), \int_{\widetilde{\gamma}_{\infty}} \tilde{\lambda}_{\infty} \neq 0, \widetilde{\gamma}_{s}:=\widetilde{F}\left(\gamma_{t}, s(t)\right) \subset B(0, R)$. Note, that the type- 1 assumption implies that $\left|\nabla^{k} \widetilde{A}\right|^{2}$ is uniformly bounded for $k \geq 0$ and all $s$. So, using the compactness of $B(0, R)$, we may assume that $\widetilde{\gamma}_{\infty}$ is the limiting curve of $\widetilde{\gamma}_{s}$ and that

$$
\begin{aligned}
& \lim _{s \rightarrow \infty} \int_{\widetilde{\gamma}_{s}} \tilde{\lambda}_{s}=\int_{\widetilde{\gamma}_{\infty}} \tilde{\lambda}_{\infty}, \\
& \lim _{s \rightarrow \infty} \int_{\widetilde{\gamma}_{s}} \widetilde{H}_{s}=\int_{\widetilde{\gamma}_{\infty}} \widetilde{H}_{\infty} .
\end{aligned}
$$


A direct computation shows that under the rescaled flow the 1-form $\widetilde{H}_{s}-2 \widetilde{\lambda}_{s}$ satisfies the evolution equation

$$
\frac{d}{d s}\left(\widetilde{H}_{s}-2 \widetilde{\lambda}_{s}\right)=d d^{\dagger}\left(\widetilde{H}_{s}-2 \widetilde{\lambda}_{s}\right)+2\left(\widetilde{H}_{s}-2 \widetilde{\lambda}_{s}\right) .
$$

Since $\widetilde{\gamma}_{s}=\widetilde{F}\left(\gamma_{t}, s(t)\right)$ and $\gamma_{t} \in e, \forall t$ we conclude

$$
\int_{\widetilde{\gamma}_{s}}\left(\widetilde{H}_{s}-2 \widetilde{\lambda}_{s}\right)=T_{\text {sing }} e^{2 s} \int_{\widetilde{\gamma}_{s_{0}}}\left(\widetilde{H}_{s_{0}}-2 \widetilde{\lambda}_{s_{0}}\right) .
$$

On the other hand

$$
\lim _{s \rightarrow \infty} \int_{\widetilde{\gamma}_{s}}\left(\widetilde{H}_{s}-2 \widetilde{\lambda}_{s}\right)=0
$$

implies

$$
\int_{\widetilde{\gamma}_{s_{0}}}\left(\widetilde{H}_{s_{0}}-2 \widetilde{\lambda}_{s_{0}}\right)=0
$$

Since $L_{0}$ is monotone we can use equation (2.4) and $\left[\widetilde{H}_{s}\right]=\left[\widetilde{H}_{s_{0}}\right]$ to obtain

$$
\left(1-\frac{1}{\varepsilon T_{\text {sing }}}\right) \int_{\widetilde{\gamma}_{s_{0}}} \widetilde{H}_{s_{0}}=0 .
$$

So either $T_{\text {sing }}=\frac{1}{\varepsilon}$ or $\int_{\widetilde{\gamma}_{s_{0}}} \widetilde{H}_{s_{0}}=0$. Since $\frac{d}{d s} \int_{\widetilde{\gamma}_{s}} \widetilde{H}_{s}=0$ and $\lim _{s \rightarrow \infty} \int_{\widetilde{\gamma}_{s}} \widetilde{H}_{s}=$ $\int_{\widetilde{\gamma}_{\infty}} \widetilde{H}_{\infty}$ we obtain $\int_{\widetilde{\gamma}_{\infty}} \widetilde{H}_{\infty}=0$ in the last case. This is a contradiction to $\widetilde{H}_{\infty}=2 \widetilde{\lambda}_{\infty}$ and $\int_{\widetilde{\gamma}_{\infty}} \widetilde{\lambda}_{\infty} \neq 0$. So $T_{\text {sing }}=\frac{1}{\varepsilon}$ and we are done.

\section{MEAN CURVATURE FLOW OF EQUiVARIANT LAGRANGIAN IMMERSIONS OF $S^{1} \times S^{n-1}$}

\subsection{The equivariant flow.}

We want to show that the Lagrangian mean curvature flow of equivariant Lagrangian immersions takes the form given in (因). Therefore, assume

$$
F(\phi, x)=(u(\phi) G(x), v(\phi) G(x))
$$

is an equivariant Lagrangian immersion as above. We will denote the coordinate on $S^{1}$ by $\phi$ and local coordinates on $S^{n-1}$ by $x^{1}, \ldots, x^{n-1}$. Latin indices $i, j, k, \ldots$ will be in the range between 1 and $n-1$, whereas greek indices $\alpha, \beta, \ldots$ are taken between 0 and $n-1$. In particular, we define coordinates $y^{\alpha}$ on $S^{1} \times S^{n-1}$ by $y^{0}:=\phi, y^{i}:=x^{i}$ for all $i \in\{1, \ldots, n-1\}$. Doubled indices will be summed according to the Einstein convention, i.e. latin indices from 1 to $n-1$ and greek indices from 0 to $n-1$.

We want to compute the metric and second fundamental form, in particular the mean curvature of these equivariant Lagrangian immersions. To this 
end, let us denote any partial derivative of $u, v$ w.r.t. $\phi$ by a prime and in addition we set $G_{i}:=\frac{\partial G}{\partial x^{i}}$ and $G_{i j}=\frac{\partial^{2} G}{\partial x^{i} \partial x^{j}}$. With this notation we get

$$
\begin{gathered}
F_{0}=\left(u^{\prime} G, v^{\prime} G\right), F_{i}=\left(u G_{i}, v G_{i}\right), \\
\nu_{0}=\left(-v^{\prime} G, u^{\prime} G\right), \nu_{i}=\left(-v G_{i}, u G_{i}\right), \\
F_{00}=\left(u^{\prime \prime} G, v^{\prime \prime} G\right), F_{0 i}=\left(u^{\prime} G_{i}, v^{\prime} G_{i}\right), F_{i j}=\left(u G_{i j}, v G_{i j}\right) .
\end{gathered}
$$

The induced metric $g_{\alpha \beta}$ and the second fundamental tensor $h_{\alpha \beta \gamma}$ on $L$ are given by $g_{\alpha \beta}=\left\langle F_{\alpha}, F_{\beta}\right\rangle$ resp. $h_{\alpha \beta \gamma}=\left\langle\nu_{\alpha}, F_{\beta \gamma}\right\rangle$. The standard metric on $S^{n-1}$ will be denoted by $\sigma_{i j}$. Thus

$$
g_{00}=\left(u^{\prime}\right)^{2}+\left(v^{\prime}\right)^{2}, g_{0 i}=0, g_{i j}=\left(u^{2}+v^{2}\right) \sigma_{i j}
$$

and

$$
h_{000}=u^{\prime} v^{\prime \prime}-v^{\prime} u^{\prime \prime}, h_{00 i}=0, h_{0 i j}=\left(u v^{\prime}-v u^{\prime}\right) \sigma_{i j}, h_{i j k}=0 .
$$

For the mean curvature form $H_{\alpha}=g^{\beta \gamma} h_{\alpha \beta \gamma}$ we obtain

$$
\begin{aligned}
& H_{0}=(n-1) \frac{u v^{\prime}-v u^{\prime}}{u^{2}+v^{2}}+\frac{u^{\prime} v^{\prime \prime}-v^{\prime} u^{\prime \prime}}{\left(u^{\prime}\right)^{2}+\left(v^{\prime}\right)^{2}}, \\
& H_{i}=0 .
\end{aligned}
$$

In particular the mean curvature vector $\vec{H}$ is given by

$$
\begin{aligned}
\vec{H} & =g^{\alpha \beta} H_{\alpha} \nu_{\beta} \\
& =g^{00} H_{0} \nu_{0} \\
& =\frac{1}{\left(u^{\prime}\right)^{2}+\left(v^{\prime}\right)^{2}}\left((n-1) \frac{u v^{\prime}-v u^{\prime}}{u^{2}+v^{2}}+\frac{u^{\prime} v^{\prime \prime}-v^{\prime} u^{\prime \prime}}{\left(u^{\prime}\right)^{2}+\left(v^{\prime}\right)^{2}}\right)\left(-v^{\prime} G, u^{\prime} G\right) .
\end{aligned}
$$

We want to rewrite the last equation in terms of objects on the curve $\gamma \subset \mathbb{C}^{*}$. If we orient $\gamma$ in the usual way, then the outer unit normal $\nu$ along $\gamma$ is given by

$$
\nu=-J\left(\frac{z^{\prime}}{\left|z^{\prime}\right|}\right) .
$$

This implies

$$
\nu=\frac{1}{\sqrt{\left(u^{\prime}\right)^{2}+\left(v^{\prime}\right)^{2}}}\left(\begin{array}{c}
v^{\prime} \\
-u^{\prime}
\end{array}\right)
$$

and

$$
\langle z, \nu\rangle=\frac{u v^{\prime}-v u^{\prime}}{\sqrt{\left(u^{\prime}\right)^{2}+\left(v^{\prime}\right)^{2}}} .
$$

The curvature $k$ of the curve $\gamma$ is determined by

$$
k=-\frac{1}{\left|z^{\prime}\right|^{2}}\left\langle z^{\prime \prime}, \nu\right\rangle=\frac{u^{\prime} v^{\prime \prime}-v^{\prime} u^{\prime \prime}}{\left(\left(u^{\prime}\right)^{2}+\left(v^{\prime}\right)^{2}\right)^{3 / 2}} .
$$

Then these equations imply

$$
\vec{H}=-\left(k+(n-1) \frac{\langle z, \nu\rangle}{|z|^{2}}\right) \frac{1}{\sqrt{\left(u^{\prime}\right)^{2}+\left(v^{\prime}\right)^{2}}}\left(v^{\prime} G,-u^{\prime} G\right) .
$$


If we project the Lagrangian mean curvature flow (MCF) to the "north-pole plane" $((1,0, \ldots, 0),(1,0, \ldots, 0)) \subset \mathbb{C}^{n}$, then the flow

$$
\frac{d}{d t} F=\vec{H}=\left(\frac{d}{d t} u G, \frac{d}{d t} v G\right)
$$

induces the flow

$$
\frac{d}{d t} z=-\left(k+(n-1) \frac{\langle z, \nu\rangle}{|z|^{2}}\right) \nu
$$

which is equation (荬).

\subsection{Singularities.}

In case of an equivariant Lagrangian immersion as above, one easily observes that the second fundamental tensor $A$ on $L$ can be completely expressed in terms of the curvature $k$ of the curves and $\frac{\left\langle\nu, e_{r}\right\rangle}{r}$ so that we obtain

Lemma 3.1. Let $\gamma \subset \mathbb{C}^{*}$ be a closed curve that evolves under the flow (困). As $t$ tends to $T_{\text {sing }}$ we must either have

$$
\limsup _{t \rightarrow T_{\text {sing }}}|k|=\infty \quad \text { or } \quad \liminf _{t \rightarrow T_{\text {sing }}} r=0 .
$$

Moreover, if $n>1$ and if there exists $\tilde{T} \in\left(0, T_{\text {sing }}\right]$ such that

$$
\liminf _{t \rightarrow \tilde{T}} r=0 \text {, }
$$

then $\tilde{T}=T_{\text {sing }}$.

Proof. At a point $\phi_{0} \in S^{1}$, where $r\left(\phi_{0}\right)=\min _{\phi \in S^{1}} r(\phi)$ we have $z= \pm r \nu$ and it follows that at such a point $\frac{\left|\left\langle\nu, e_{r}\right\rangle\right|}{r}=\frac{1}{r}$. If $n>1$ and $r$ tends to zero somewhere, then the corresponding equivariant Lagrangian map fails to be an immersion as $t \rightarrow \tilde{T}$.

In view of the last lemma we distinguish the following three cases:

$$
\begin{array}{lll}
\limsup _{t \rightarrow T_{\text {sing }}}|k|=\infty & \text { and } & \liminf _{t \rightarrow T_{\text {sing }}} r=0, \\
\limsup _{t \rightarrow T_{\text {sing }}}|k|=\infty & \text { and } & \liminf _{t \rightarrow T_{\text {sing }}} r>0, \\
\limsup _{t \rightarrow T_{\text {sing }}}|k|<\infty & \text { and } & \liminf _{t \rightarrow T_{\text {sing }}} r=0 .
\end{array}
$$

Remark 3.2. A priori we cannot exclude a singularity of type $(C 3)$ (except in the case $n=1)$. One can prove that in case $(C 3)$ the curves converge in the $C^{\infty}$-topology to a smooth limit curve $\gamma$ passing through the origin as $t \rightarrow T_{\text {sing. }}$. The singularity for the corresponding Lagrangian immersions then occurs, because in the limit $t \rightarrow T_{\text {sing }}$ the equivariant maps $F: S^{1} \times$ $S^{n-1} \rightarrow \mathbb{C}^{n}$ fail to be an immersion. We thus call a singularity of type (C3) a fake singularity. The next lemma states that the quantity $\frac{\left\langle\nu, e_{r}\right\rangle}{r}$ and 
therefore also the equivariant Lagrangian mean curvature flow would still make sense for those curves.

Lemma 3.3. Let $z: S^{1} \rightarrow \mathbb{C}$ be a smooth regular curve. Assume $\phi_{0} \in S^{1}$ is a point with $z\left(\phi_{0}\right)=0$. Then we have

$$
\lim _{\phi \rightarrow \phi_{0}} \frac{\langle z(\phi), \nu(\phi)\rangle}{|z(\phi)|^{2}}=\frac{1}{2} k\left(\phi_{0}\right)
$$

where $k\left(\phi_{0}\right)$ denotes the curvature of the curve at $z\left(\phi_{0}\right)=0$ and $\nu(\phi)$ is the inward pointing unit normal.

Proof. Let $(x, y)$ be cartesian coordinates for $\mathbb{C}$ such that at the origin the $x$-axis is tangent to our curve $\gamma:=z\left(S^{1}\right)$. Then locally, say on an interval $(-\epsilon, \epsilon)$, we can express $\gamma$ as a graph over the $x$-axis, i.e. w.l.o.g. we may assume that after a reparameterization our curve is locally given by $z(x)=$ $(x, y(x))$ with a smooth function $y:(-\epsilon, \epsilon) \rightarrow \mathbb{R}$. It is sufficient to prove the lemma for real analytic functions $y$. Let

$$
y(x)=\sum_{k=0}^{\infty} \frac{x^{k}}{k !} y^{(k)}(0)
$$

be the Taylor expansion of $y$ around 0 . For $x \neq 0$ we have

$$
\frac{\langle z, \nu\rangle}{|z|^{2}}=\frac{x y^{\prime}-y}{\left(x^{2}+y^{2}\right) \sqrt{1+\left(y^{\prime}\right)^{2}}}=\frac{\frac{y^{\prime}}{x}-\frac{y}{x^{2}}}{\left(1+\left(\frac{y}{x}\right)^{2}\right) \sqrt{1+\left(y^{\prime}\right)^{2}}} .
$$

Since $y(0)=0$ we see that

$$
\lim _{x \rightarrow 0} \frac{y^{2}}{x^{2}}=\left(y^{\prime}(0)\right)^{2} .
$$

If we take into account the Taylor expansion we obtain

$$
\frac{1}{x^{2}}\left(y-x y^{\prime}\right)=\sum_{k=2}^{\infty} x^{k-2} y^{(k)}(0)\left(\frac{1}{k !}-\frac{1}{(k-1) !}\right)
$$

and this implies

$$
\lim _{x \rightarrow 0} \frac{1}{x^{2}}\left(y-x y^{\prime}\right)=-\frac{1}{2} y^{\prime \prime}(0)
$$

so that

$$
\lim _{x \rightarrow 0} \frac{\langle z(x), \nu(x)\rangle}{|z(x)|^{2}}=\frac{y^{\prime \prime}(0)}{2\left(1+\left(y^{\prime}(0)\right)^{2}\right)^{3 / 2}}=\frac{1}{2} k(0) .
$$

This proves the lemma.

Remark 3.4. One has to be a bit careful here. If $z: S^{1} \times\left[0, T_{\text {sing }}\right) \rightarrow \mathbb{C}^{*}$ is a smooth family of curves with $\liminf _{t \rightarrow T_{\text {sing }}} r=0$, then clearly

$$
\limsup _{t \rightarrow T_{\text {sing }}} \frac{\left|\left\langle\nu, e_{r}\right\rangle\right|}{r}=\infty \text {. }
$$


But if a smooth limit curve (passing through the origin) exists, then lemma 3.3 does not prove that the curvature $k$ blows up at the origin (but $|A|$ does for $\left.t \rightarrow T_{\text {sing }}\right)$. This shows that (函) would also be well defined for a smooth curve passing through the origin and a solution of (函) might exist longer than the corresponding solution for $(M C F)$. On the other hand, it is likely that fake singularities will not occur at all under the mean curvature flow.

\subsection{Embeddedness.}

The next question we address is, if the embeddedness of the profile curve $\gamma \subset \mathbb{C}^{*}$ will also imply the embeddedness of the equivariant Lagrangian submanifold associated to $\gamma$.

Lemma 3.5. Let $\gamma \subset \mathbb{C}^{*}$ be an embedded curve given by an embedding $z: S^{1} \rightarrow \mathbb{C}^{*}$. The image $L:=F\left(S^{1} \times S^{n-1}\right), F(\phi, p)=(u(\phi) p, v(\phi) p)$ is an embedded Lagrangian submanifold, if and only if one of the following conditions is valid: (i) the curve $\gamma$ satisfies $\gamma \cap(-\gamma)=\emptyset$, (ii) $\gamma$ is pointsymmetric, i.e. it satisfies $\gamma=-\gamma$. In the latter case, $F$ is a double covering of $L$.

Proof. Let $\left(\phi_{1}, p_{1}\right),\left(\phi_{2}, p_{2}\right) \in S^{1} \times S^{n-1}$ to points with $F\left(\phi_{1}, p_{1}\right)=F\left(\phi_{2}, p_{2}\right)$. Then either $p_{1}=p_{2}, z\left(\phi_{1}\right)=z\left(\phi_{2}\right)$ which, in view of the embeddedness of $\gamma$ implies $\phi_{1}=\phi_{2}$, or $p_{1}=-p_{2}, z\left(\phi_{1}\right)=-z\left(\phi_{2}\right)$. So if $\gamma \cap(-\gamma) \neq \emptyset$ and $\gamma \cap(-\gamma) \neq \gamma$, then $L$ cannot be embedded. The rest of the lemma is clear.

Lemma 3.6. If the initial curve is embedded, then this remains true for all $t \in\left[0, T_{\text {sing }}\right)$.

Proof. This is well-known for $n=1$. Assume $n>1$ and that the curve touches itself for the first time at $t_{0}<T_{\text {sing. }}$. Let $\phi_{1}, \phi_{2} \in S^{1}$ be two distinct points with $z\left(\phi_{1}\right)=z\left(\phi_{2}\right)$. Since $t_{0}<T_{\text {sing }}$ we must have $|z|>0$ on $S^{1}$ (Lemma 3.1). It suffices to prove that $\left\langle f\left(\phi_{1}\right) \nu\left(\phi_{1}\right)-f\left(\phi_{2}\right) \nu(\phi 2), \nu\left(\phi_{1}\right)-\right.$ $\left.\nu\left(\phi_{2}\right)\right\rangle \leq 0$ because this contradicts the strong maximum principle. The curvatures at $\phi_{1}$ and $\phi_{2}$ must satisfy $k\left(\phi_{1}\right)+k\left(\phi_{2}\right) \leq 0$. Moreover, we have

$\nu\left(\phi_{1}\right)=-\nu\left(\phi_{2}\right), z\left(\phi_{1}\right)=z\left(\phi_{2}\right)$ and $\frac{\left\langle\nu, e_{r}\right\rangle}{r}\left(\phi_{1}\right)=-\frac{\left\langle\nu, e_{r}\right\rangle}{r}\left(\phi_{2}\right)$. Therefore one has

$$
\begin{aligned}
& \left\langle f\left(\phi_{1}\right) \nu\left(\phi_{1}\right)-f\left(\phi_{2}\right) \nu(\phi 2), \nu\left(\phi_{1}\right)-\nu\left(\phi_{2}\right)\right\rangle \\
& =2\left(k\left(\phi_{1}\right)+(n-1) \frac{\left\langle\nu, e_{r}\right\rangle}{r}\left(\phi_{1}\right)+k\left(\phi_{2}\right)+(n-1) \frac{\left\langle\nu, e_{r}\right\rangle}{r}\left(\phi_{2}\right)\right) \\
& =2\left(k\left(\phi_{1}\right)+k\left(\phi_{2}\right)\right) \leq 0 .
\end{aligned}
$$

Remark 3.7. The lemma does not imply that the curves stay embedded as

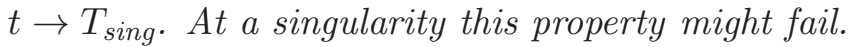




\subsection{Tamed curves.}

Definition 3.8. A curve $\gamma \subset \mathbb{C}^{*}$ will be called tamed, if the quantity

$$
f:=k+(n-1) \frac{\langle z, \nu\rangle}{|z|^{2}}
$$

is positive for all $p \in \gamma$.

In the sequel we will set

$$
r:=|z|, \quad e_{r}:=\frac{z}{|z|}
$$

so that

$$
f=k+(n-1) \frac{\left\langle\nu, e_{r}\right\rangle}{r} .
$$

Lemma 3.9. Let $\gamma \subset \mathbb{C}^{*}$ be a smooth, closed curve. Then there exists at least one point $p \in \gamma$ where

$$
f \geq \frac{n}{r}>0 .
$$

Proof. Let $z: S^{1} \rightarrow \mathbb{C}^{*}$ be a smooth immersion of $\gamma$ and $\phi \in S^{1}$ a point where $r$ assumes its maximum. By definition of the outer unit normal we have at this point $z=r \nu$. Since at $\phi$ we must have $\Delta r^{2}=\Delta|z|^{2} \leq 0$, the identity $\Delta z=-k \nu$ gives

$$
0 \geq \Delta r^{2}=2(1-k\langle z, \nu\rangle)=2(1-k r) .
$$

Therefore

$$
f=k+(n-1) \frac{\left\langle\nu, e_{r}\right\rangle}{r} \geq \frac{1}{r}+(n-1) \frac{1}{r}=\frac{n}{r} .
$$

From this and equation (3.3) follows:

Corollary 3.10. A curve $\gamma \subset \mathbb{C}^{*}$ is tamed if and only if the corresponding equivariant Lagrangian immersion of $S^{1} \times S^{n-1}$ into $\mathbb{C}^{n}$ has a nowhere vanishing mean curvature vector field $\vec{H}$.

Next we will compute several evolution equations related to (函. From [18] we first obtain the equations:

\section{Lemma 3.11.}

$$
\begin{aligned}
\frac{d}{d t} d \mu & =-k f d \mu \\
\frac{d}{d t} \nu & =\nabla f \\
\frac{d}{d t} k & =\Delta f+f k^{2},
\end{aligned}
$$

where $d \mu$ denotes the induced volume form on $\gamma$. 
Lemma 3.12. The evolution equation for $f$ is given by

$$
\frac{d}{d t} f=\Delta f+\frac{n-1}{r}\left\langle\nabla f, e_{r}\right\rangle+f\left(k^{2}+\frac{n-1}{r^{2}}\left(2\left\langle\nu, e_{r}\right\rangle^{2}-1\right)\right) .
$$

Proof. We have

$$
\frac{d}{d t} \frac{\langle z, \nu\rangle}{r^{2}}=\frac{-f+\langle z, \nabla f\rangle}{r^{2}}+\frac{2\langle z, \nu\rangle^{2} f}{r^{4}}
$$

and then with (3.6)

$$
\frac{d}{d t} f=\Delta f+f k^{2}+(n-1)\left(\frac{-f+\langle z, \nabla f\rangle}{r^{2}}+\frac{2\langle z, \nu\rangle^{2} f}{r^{4}}\right) .
$$

This and the maximum principle implies:

Corollary 3.13. If the initial curve $\gamma_{0} \subset \mathbb{C}^{*}$ is tamed, then it stays tamed for $t \in\left[0, T_{\text {sing }}\right)$.

If we take into account Corollary 3.10, then also

Corollary 3.14. If the initial equivariant Lagrangian immersion L satisfies $\vec{H} \neq 0$ for all $x \in L$, then this remains true for all $t \in\left[0, T_{\text {sing }}\right)$.

\subsection{Monotonicity.}

We want to find the condition when an equivariant Lagrangian immersion with a profile curve $\gamma \subset \mathbb{C}^{*}$ becomes monotone. Since we have already computed the mean curvature form in (3.1) and (3.2), we need to compute $F^{*} \lambda$ only.

Since

$$
\left(F^{*} \lambda\right)_{\alpha}=\frac{1}{2}\left\langle J F, F_{\alpha}\right\rangle
$$

and

$$
\begin{aligned}
J F & =(-v G, u G), \\
F_{\alpha} & =\left(u_{\alpha} G+u G_{\alpha}, v_{\alpha} G+v G_{\alpha}\right), \\
|G|^{2} & =1 \\
\left\langle G, G_{\alpha}\right\rangle & =0
\end{aligned}
$$

we obtain:

$$
\left(F^{*} \lambda\right)_{\alpha}=\frac{1}{2}\left(u v_{\alpha}-v u_{\alpha}\right)
$$


i.e.

$$
\begin{array}{r}
\left(F^{*} \lambda\right)_{0}=\frac{1}{2}\left(u v^{\prime}-v u^{\prime}\right), \\
\left(F^{*} \lambda\right)_{i}=0, \quad \forall i=1, \ldots, n .
\end{array}
$$

From equations (3.1), (3.2), (3.8) and (3.9) we see that $[H]=\varepsilon\left[F^{*} \lambda\right]$, if and only if

$$
\int_{\gamma} H=\varepsilon \int_{\gamma} z^{*} \lambda
$$

Now, since by (3.1) and (3.8)

$$
H_{0}=f d \mu, \quad\left(F^{*} \lambda\right)_{0}=\frac{1}{2}\langle z, \nu\rangle d \mu
$$

we obtain

Lemma 3.15. An equivariant Lagrangian immersion is monotone with monotonicity constant $\varepsilon$, if and only if on the profile curve $z: S^{1} \rightarrow \mathbb{C}^{*}$ we have

$$
\int_{S^{1}} f d \mu=\frac{\varepsilon}{2} \int_{S^{1}}\langle z, \nu\rangle d \mu
$$

If we define the symplectic area $A(z)$ of a regular, smooth curve $z: S^{1} \rightarrow \mathbb{C}^{*}$ by

$$
A(z):=\int_{S^{1}} z^{*} \lambda
$$

then the computations above show

$$
A(z)=\frac{1}{2} \int_{S^{1}}\langle z, \nu\rangle d \mu .
$$

Moreover, the next lemma gives a geometric interpretation of $\int_{S^{1}} f d \mu$ as well.

Lemma 3.16. Suppose $z: S^{1} \rightarrow \mathbb{C}^{*}$ is a regular, smooth curve. Let $\operatorname{wind}_{0}(z)$ and $\operatorname{rot}(z)$ denote the winding number of $z$ around the origin resp. the rotation number. Then

$$
\int_{S^{1}} f d \mu=2 \pi\left(\operatorname{rot}(z)+(n-1) \operatorname{wind}_{0}(z)\right) .
$$

Proof. The vector field $Z(z):=z$ satisfies

$$
\operatorname{div}(Z)=2 \text { and } \operatorname{div}\left(\frac{Z}{|z|^{2}}\right)=0, \text { for } z \neq 0
$$

Stokes theorem gives

$$
A(z)=\frac{1}{2} \int_{S^{1}}\langle z, \nu\rangle d \mu
$$


and

Since

$$
2 \pi \operatorname{wind}_{0}(z)=\int_{S^{1}}\left\langle\frac{z}{|z|^{2}}, \nu\right\rangle d \mu
$$

$$
f=k+(n-1)\left\langle\frac{z}{|z|^{2}}, \nu\right\rangle
$$

and

$$
\int_{S^{1}} k d \mu=2 \pi \operatorname{rot}(z)
$$

the proof follows.

From Lemma 3.15 and Lemma 3.16 we immediately obtain:

Corollary 3.17. Suppose $z: S^{1} \rightarrow \mathbb{C}^{*}$ is a regular, smooth curve with $A(z) \neq 0$ and $\left(\operatorname{rot}(z)+(n-1) \operatorname{wind}_{0}(z)\right) / A(z)>0$. Then the corresponding equivariant Lagrangian immersion is monotone with monotonicity constant

$$
\varepsilon=\frac{2 \pi\left(\operatorname{rot}(z)+(n-1) \operatorname{wind}_{0}(z)\right)}{A(z)}>0 .
$$

\subsection{The symplectic area formula.}

Theorem 3.18. Suppose $z: S^{1} \times\left[0, T_{\text {sing }}\right) \rightarrow \mathbb{C}^{*}$ is a smooth solution of (困). Then the symplectic area $A\left(z_{t}\right)$ of $z_{t}:=z(\cdot, t): S^{1} \rightarrow \mathbb{C}^{*}$, satisfies the following equation

$$
A\left(z_{t}\right)=A\left(z_{0}\right)-2 \pi\left(\operatorname{rot}\left(z_{0}\right)+(n-1) \operatorname{wind}_{0}\left(z_{0}\right)\right) t .
$$

Proof. Since $\frac{d}{d t}\langle z, \nu\rangle=-f+\langle\nabla f, z\rangle$ and $\frac{d}{d t} d \mu=-f k d \mu$ we obtain

$$
\frac{d}{d t} A(z)=\frac{1}{2} \int_{S^{1}}(\langle\nabla f, z\rangle-f-\langle z, \nu\rangle f k) d \mu
$$

and with partial integration

$$
\frac{d}{d t} A(z)=\frac{1}{2} \int_{S^{1}}(-f(1-k\langle z, \nu\rangle)-f-\langle z, \nu\rangle f k) d \mu=-\int_{S^{1}} f d \mu .
$$

By Lemma 3.16 we thus conclude

$$
\frac{d}{d t} A\left(z_{t}\right)=-2 \pi\left(\operatorname{rot}\left(z_{t}\right)+(n-1) \operatorname{wind}_{0}\left(z_{t}\right)\right)
$$

The rotation number is always invariant under homotopies so that $\operatorname{rot}\left(z_{t}\right)=$ $\operatorname{rot}\left(z_{0}\right)$. For $n>1$, Lemma 3.1 implies $|z|>0$ on [0, $\left.T_{\text {sing }}\right)$. Thus, in case $n>1, \operatorname{wind}_{0}\left(z_{t}\right)=$ wind $_{0}\left(z_{0}\right)$. This proves the theorem.

Corollary 3.19. Suppose $z: S^{1} \times\left[0, T_{\text {sing }}\right) \rightarrow \mathbb{C}^{*}$ is a smooth solution of (困) and that $z_{0}: S^{1} \rightarrow \mathbb{C}^{*}$ is an embedding. Then

$$
T_{\text {sing }} \leq \frac{A\left(z_{0}\right)}{2 \pi\left(\operatorname{rot}\left(z_{0}\right)+(n-1) \text { wind }_{0}\left(z_{0}\right)\right)} .
$$


Proof. First note, that this does not follow from Theorem [1.6 since the equivariant Lagrangian submanifold need not be embedded. On the other hand an embedded closed curve satisfies $\operatorname{rot}\left(z_{0}\right)=1$ and wind ${ }_{0}\left(z_{0}\right) \in\{0,1\}$ depending on whether the curve encloses the origin or not. Since $A\left(z_{0}\right)$ is the area of the enclosed region of $z_{0}$ we see that $A\left(z_{t}\right)>0$ for all $t \in\left[0, T_{\text {sing }}\right)$ because the curves stay embedded. The symplectic area formula above shows that this can be true only as long as

$$
T_{\text {sing }} \leq \frac{A\left(z_{0}\right)}{2 \pi\left(\operatorname{rot}\left(z_{0}\right)+(n-1) \operatorname{wind}_{0}\left(z_{0}\right)\right)} .
$$

Note also, that the equivariant Lagrangian submanifold for $z_{0}$ is monotone with monotonicity constant

$$
\varepsilon=\frac{2 \pi\left(\operatorname{rot}\left(z_{0}\right)+(n-1) \operatorname{wind}_{0}\left(z_{0}\right)\right)}{A\left(z_{0}\right)}
$$

so that we have

$$
T_{\mathrm{emb}}=T_{\text {sing }} \leq \frac{1}{\varepsilon} .
$$

\subsection{Selfsimilar solutions.}

Like for the monotonicity, the equation for self-similar solutions (1.4) takes a much simpler form in the equivariant case, namely

$$
f=\frac{\varepsilon}{2}\langle z, \nu\rangle \text {. }
$$

Henri Anciaux 11 classified all solutions of (3.12). He noticed that - in contrast to the curve-shortening flow - not all solutions of (3.12) are convex. On the other hand, in view of Corollary 3.10 tameness is a much more natural condition than convexity and by (3.12) any tamed solution is also starshaped. Indeed, as the next theorem shows, all solutions of (3.12) with $\varepsilon \neq 0$ are tamed and starshaped.

Theorem 3.20. Assume $z$ is a self-similar expanding, or contracting solution of

$$
\frac{d}{d t} z=-f \nu
$$

i.e. there exists a constant $\varepsilon \neq 0$ such that

$$
f=\frac{\varepsilon}{2}\langle z, \nu\rangle \text {. }
$$

Then the quantity

$$
p:=f e^{-\frac{\varepsilon}{4}|z|^{2}}|z|^{n-1}
$$

is constant along the curve and the curve is tamed and starshaped. 
Proof. The Weingarten equation implies

$$
\nabla\langle z, \nu\rangle=\frac{1}{2} k \nabla|z|^{2}
$$

In view of (1.10) and (3.12) we obtain

$$
k=\left(1-2 \frac{n-1}{\varepsilon|z|^{2}}\right) f .
$$

If we take the gradient of both sides in (3.12), then we obtain

$$
\begin{aligned}
\nabla f & =\frac{\varepsilon}{2} \nabla\langle z, \nu\rangle \\
& =\left(\frac{\varepsilon}{4}-\frac{n-1}{2|z|^{2}}\right) f \nabla|z|^{2},
\end{aligned}
$$

where we have used (3.13) and (3.14) in the last step. We claim that the quantity

$$
p:=f e^{-\frac{\varepsilon}{4}|z|^{2}}|z|^{n-1}
$$

is constant on any solution of (1.10). The quantity $p$ is differentiable whenever $z \neq 0$. By (3.15) the gradient of $p$ at those points is

$$
\begin{aligned}
\nabla p & =\left(\frac{\varepsilon}{4}-\frac{n-1}{2|z|^{2}}\right) p \nabla|z|^{2}-\frac{\varepsilon}{4} p \nabla|z|^{2}+\frac{n-1}{|z|} p \nabla|z| \\
& =0 .
\end{aligned}
$$

The constant $p$ can only be zero, if $f \equiv 0$ which is equivalent to $z$ being a segment of a line passing through the origin. In particular, any solution of (3.12) passing through the origin must be a line segment.

For a curve different from a line there exists always one point, where $\langle z, \nu\rangle>$ 0 . Consequently, by (3.12), $p$ must be a positive constant and it also follows that any solution $z: S^{1} \rightarrow \mathbb{C}^{*}$ of (3.12) is starshaped w.r.t. the origin and satisfies $f>0$.

Remark 3.21. In particular, by Corollary 3.10, the corresponding selfsimilarly evolving equivariant Lagrangian immersions in $\mathbb{C}^{n}$ all have nowhere vanishing mean curvature vector $\vec{H}$. Moreover, as has been noted earlier by Anciaux 1, for $n=1$ the curves are all convex, whereas for $n>1$ we have

$$
\begin{aligned}
& k>0, \quad \text { for }|z|>\sqrt{\frac{2(n-1)}{\varepsilon}} \\
& k=0, \quad \text { for }|z|=\sqrt{\frac{2(n-1)}{\varepsilon}} \\
& k<0, \quad \text { for }|z|<\sqrt{\frac{2(n-1)}{\varepsilon}} .
\end{aligned}
$$




\subsection{Starshapedness.}

A curve $z: S^{1} \rightarrow \mathbb{C}^{*}$ will be called starshaped, if $\langle\nu, z\rangle>0$ everywhere. In this section we want to show that starshapedness is preserved as long as $r=|z|>0$. To this end we need some evolution equations and the following lemma which will be used several times in our computations

Lemma 3.22. On any curve $\gamma \in \mathbb{C}^{*}$ we have

$$
\begin{aligned}
1 & =|\nabla r|^{2}+\left\langle\nu, e_{r}\right\rangle^{2}, \\
\Delta r & =\left\langle\nu, e_{r}\right\rangle\left(\frac{\left\langle\nu, e_{r}\right\rangle}{r}-k\right), \\
\nabla\left\langle\nu, e_{r}\right\rangle & =\left(k-\frac{\left\langle\nu, e_{r}\right\rangle}{r}\right) \nabla r .
\end{aligned}
$$

Proof. We have

$$
z=\frac{\left\langle z, z^{\prime}\right\rangle}{\left|z^{\prime}\right|^{2}} z^{\prime}+\langle z, \nu\rangle \nu
$$

and therefore

$$
r^{2}=|z|^{2}=\frac{\left|\left\langle z, z^{\prime}\right\rangle\right|^{2}}{\left|z^{\prime}\right|^{2}}+\langle z, \nu\rangle^{2}=\left|\nabla \frac{r^{2}}{2}\right|^{2}+\langle z, \nu\rangle^{2}
$$

which proves the first equation. The second equation then follows from the first and Gauß' equation $\Delta z=-k \nu$. The last equation follows from $\nu^{\prime}=k z^{\prime}$.

Next we want to compute the evolution equations for $r$ and $\left\langle\nu, e_{r}\right\rangle$.

Lemma 3.23. The following equations are valid

$$
\begin{aligned}
\frac{d}{d t} r= & \Delta r+\frac{n-1}{r}\left\langle\nabla r, e_{r}\right\rangle-\frac{1}{r}\left\langle\nu, e_{r}\right\rangle^{2}-(n-1) \frac{1}{r} \\
\frac{d}{d t}\left\langle\nu, e_{r}\right\rangle & =\Delta\left\langle\nu, e_{r}\right\rangle+\frac{n-1}{r}\left\langle\nabla\left\langle\nu, e_{r}\right\rangle, e_{r}\right\rangle \\
& -2 n \frac{\left\langle\nu, e_{r}\right\rangle}{r^{2}}\left(1-\left\langle\nu, e_{r}\right\rangle^{2}\right)+\left(k-\frac{\left\langle\nu, e_{r}\right\rangle}{r}\right)^{2}\left\langle\nu, e_{r}\right\rangle
\end{aligned}
$$

Proof. We first observe that

$$
\frac{d}{d t} r=\frac{1}{r}\left\langle z, \frac{d}{d t} z\right\rangle=-f\left\langle\nu, e_{r}\right\rangle,
$$

so that in view of (3.16) and $\left\langle\nabla r, e_{r}\right\rangle=|\nabla r|^{2}$

$$
\frac{d}{d t} r=-k\left\langle\nu, e_{r}\right\rangle+\frac{n-1}{r}\left\langle\nabla r, e_{r}\right\rangle-\frac{n-1}{r} .
$$


On the other hand, by (3.17) the Laplacian of $r$ is given by

$$
\Delta r=\left\langle\nu, e_{r}\right\rangle\left(\frac{\left\langle\nu, e_{r}\right\rangle}{r}-k\right)
$$

Hence

$$
\frac{d}{d t} r=\Delta r+\frac{n-1}{r}\left\langle\nabla r, e_{r}\right\rangle-\frac{1}{r}\left\langle\nu, e_{r}\right\rangle^{2}-(n-1) \frac{1}{r}
$$

For the evolution equation of $\left\langle\nu, e_{r}\right\rangle$ we compute

$$
\begin{aligned}
\frac{d}{d t}\left\langle\nu, e_{r}\right\rangle= & \left\langle\nabla f, e_{r}\right\rangle-\frac{\left\langle\nu, e_{r}\right\rangle}{r^{2}}\left\langle z, \frac{d}{d t} z\right\rangle-\frac{f}{r} \\
= & \left\langle\nabla f, e_{r}\right\rangle-\frac{f}{r}\left(1-\left\langle\nu, e_{r}\right\rangle^{2}\right) \\
= & \left\langle\nabla k, e_{r}\right\rangle+\frac{n-1}{r}\left\langle\nabla\left\langle\nu, e_{r}\right\rangle, e_{r}\right\rangle \\
& -\left(\frac{f}{r}+(n-1) \frac{\left\langle\nu, e_{r}\right\rangle}{r^{2}}\right)\left(1-\left\langle\nu, e_{r}\right\rangle^{2}\right) .
\end{aligned}
$$

To compute the Laplacian we use (3.17) and (3.18) to derive

$$
\begin{aligned}
\Delta\left\langle\nu, e_{r}\right\rangle= & \left\langle\nabla k, e_{r}\right\rangle-\left(k-\frac{\left\langle\nu, e_{r}\right\rangle}{r}\right)^{2}\left\langle\nu, e_{r}\right\rangle \\
& -\frac{1-\left\langle\nu, e_{r}\right\rangle^{2}}{r}\left(k-\frac{\left\langle\nu, e_{r}\right\rangle}{r}\right)+\frac{\left\langle\nu, e_{r}\right\rangle}{r^{2}}\left(1-\left\langle\nu, e_{r}\right\rangle^{2}\right) .
\end{aligned}
$$

Combining these two equations we get

$$
\begin{aligned}
\frac{d}{d t}\left\langle\nu, e_{r}\right\rangle= & \Delta\left\langle\nu, e_{r}\right\rangle+\frac{n-1}{r}\left\langle\nabla\left\langle\nu, e_{r}\right\rangle, e_{r}\right\rangle \\
& -\left(\frac{f}{r}+n \frac{\left\langle\nu, e_{r}\right\rangle}{r^{2}}\right)\left(1-\left\langle\nu, e_{r}\right\rangle^{2}\right) \\
& +\left(k-\frac{\left\langle\nu, e_{r}\right\rangle}{r}\right)^{2}\left\langle\nu, e_{r}\right\rangle+\frac{1-\left\langle\nu, e_{r}\right\rangle^{2}}{r}\left(k-\frac{\left\langle\nu, e_{r}\right\rangle}{r}\right) \\
= & \Delta\left\langle\nu, e_{r}\right\rangle+\frac{n-1}{r}\left\langle\nabla\left\langle\nu, e_{r}\right\rangle, e_{r}\right\rangle \\
& -2 n \frac{\left\langle\nu, e_{r}\right\rangle}{r^{2}}\left(1-\left\langle\nu, e_{r}\right\rangle^{2}\right)+\left(k-\frac{\left\langle\nu, e_{r}\right\rangle}{r}\right)^{2}\left\langle\nu, e_{r}\right\rangle .
\end{aligned}
$$

Corollary 3.24. If the initial curve $\gamma_{0} \subset \mathbb{C}^{*}$ is starshaped, i.e. if

$$
\left\langle\nu, e_{r}\right\rangle>0
$$

for all $p \in \gamma_{0}$, then this remains true as long as a smooth solution of (困) exists in $\mathbb{C}^{*}$. 
Proof. Suppose a smooth solution in $\mathbb{C}^{*}$ exists on a time interval $\left[0, t_{1}\right)$ and that $0<t_{0}<t_{1}$ is some fixed time. There exists a constant $\lambda>0$ depending on $t_{0}$ such that

$$
\frac{2 n}{r^{2}}\left(1-\left\langle\nu, e_{r}\right\rangle^{2}\right)-\left(k-\frac{\left\langle\nu, e_{r}\right\rangle}{r}\right)^{2} \leq \lambda
$$

holds on the compact time interval $\left[0, t_{0}\right]$. Then by (3.20) we can estimate for $t \in\left[0, t_{0}\right]$

$$
\frac{d}{d t}\left\langle\nu, e_{r}\right\rangle \geq \Delta\left\langle\nu, e_{r}\right\rangle+\frac{n-1}{r}\left\langle\nabla\left\langle\nu, e_{r}\right\rangle, e_{r}\right\rangle-\lambda\left\langle\nu, e_{r}\right\rangle
$$

and the maximum principle implies that

$$
\left\langle\nu, e_{r}\right\rangle \geq\left(\inf _{\gamma_{0}}\left\langle\nu, e_{r}\right\rangle\right) e^{-\lambda t}, \quad \forall t \in\left[0, t_{0}\right]
$$

\section{Remark 3.25.}

(a) Corollary 3.24 is sharp in the following sense: If $n=1$, i.e. if we consider the usual curve shortening flow, and if the initial curve is a circle of some radius including the origin but not centered at the origin, then this remains starshaped as long as the origin is contained in the shrinking circles. As soon as one circle touches the origin, starshapedness will fail.

(b) If $n>1$, then by Lemma 3.1 a smooth solution of (函) with initial curve $\gamma_{0} \in \mathbb{C}^{*}$ is contained in $\mathbb{C}^{*}$ for any $t \in\left[0, T_{\text {sing }}\right)$.

We can prove even stronger estimates:

Lemma 3.26. If the initial curve $\gamma_{0} \subset \mathbb{C}^{*}$ is starshaped, then for any $p \geq 2$ there exists a constant $\epsilon_{p}>0$ such that

$$
r^{-p}\left\langle\nu, e_{r}\right\rangle \geq \epsilon_{p}
$$

holds true as long as a smooth solution of (困) exists in $\mathbb{C}^{*}$.

Proof. First we compute the evolution equation of $m:=r^{-p}\left\langle\nu, e_{r}\right\rangle$. Therefore, in general the following computations are valid only as long as $r>0$. According to Corollary 3.24 and Remark 3.25 this quantity is well defined for $t \in\left[0, T_{\text {sing }}\right)$, if $n>1$ and in case $n=1$ as long as $r>0$. Lemma B.23 
implies

$$
\begin{aligned}
\frac{d}{d t} m= & \left\langle\nu, e_{r}\right\rangle\left\{\Delta r^{-p}+\frac{n-1}{r}\left\langle\nabla r^{-p}, e_{r}\right\rangle+p(p+2) r^{-p-2}\left\langle\nu, e_{r}\right\rangle^{2}\right. \\
& \left.-p(p-n+2) r^{-p-2}\right\}+r^{-p}\left\{\Delta\left\langle\nu, e_{r}\right\rangle\right. \\
& +\frac{n-1}{r}\left\langle\nabla\left\langle\nu, e_{r}\right\rangle, e_{r}\right\rangle-2 n \frac{\left\langle\nu, e_{r}\right\rangle}{r^{2}}\left(1-\left\langle\nu, e_{r}\right\rangle^{2}\right) \\
& \left.+\left(k-\frac{\left\langle\nu, e_{r}\right\rangle}{r}\right)^{2}\left\langle\nu, e_{r}\right\rangle\right\} .
\end{aligned}
$$

The Laplacian of $m$ is given by

$$
\begin{aligned}
\Delta m= & r^{-p} \Delta\left\langle\nu, e_{r}\right\rangle+\left\langle\nu, e_{r}\right\rangle \Delta r^{-p}+2\left\langle\nabla\left\langle\nu, e_{r}\right\rangle, \nabla r^{-p}\right\rangle \\
= & r^{-p} \Delta\left\langle\nu, e_{r}\right\rangle+\left\langle\nu, e_{r}\right\rangle \Delta r^{-p} \\
& -2 p r^{-p-1}\left(k-\frac{\left\langle\nu, e_{r}\right\rangle}{r}\right)\left(1-\left\langle\nu, e_{r}\right\rangle^{2}\right),
\end{aligned}
$$

where we have used (3.16) and (3.18). Therefore it follows

$$
\begin{aligned}
\frac{d}{d t} m= & \Delta m+\frac{n-1}{r}\left\langle\nabla m, e_{r}\right\rangle+2 p r^{-p-1}\left(k-\frac{\left\langle\nu, e_{r}\right\rangle}{r}\right)\left(1-\left\langle\nu, e_{r}\right\rangle^{2}\right) \\
& +m\left\{-\frac{p^{2}+2 p+2 n}{r^{2}}\left(1-\left\langle\nu, e_{r}\right\rangle^{2}\right)+\frac{n p}{r^{2}}+\left(k-\frac{\left\langle\nu, e_{r}\right\rangle}{r}\right)^{2}\right\} .
\end{aligned}
$$

We will show that $m$ cannot admit a decreasing positive minimum. At a minimum of $m$ we have $\nabla m=0, \Delta m \geq 0$. From

$$
\nabla m=r^{-p}\left(k-(p+1) \frac{\left\langle\nu, e_{r}\right\rangle}{r}\right) \nabla r=0
$$

we conclude, that at such a point either $\nabla r=0$, or $k-\frac{\left\langle\nu, e_{r}\right\rangle}{r}=p \frac{\left\langle\nu, e_{r}\right\rangle}{r}$. In the first case we also have $1-\left\langle\nu, e_{r}\right\rangle^{2}=0$ and then

$$
\frac{d}{d t} m \geq m\left\{\frac{n p}{r^{2}}+\left(k-\frac{\left\langle\nu, e_{r}\right\rangle}{r}\right)^{2}\right\}>0 .
$$

In the second case, we substitute $k-\frac{\left\langle\nu, e_{r}\right\rangle}{r}$ and obtain

$$
\begin{aligned}
\frac{d}{d t} m & \geq m\left\{\frac{p^{2}-2 p-2 n}{r^{2}}\left(1-\left\langle\nu, e_{r}\right\rangle^{2}\right)+\frac{n p}{r^{2}}+p^{2} \frac{\left\langle\nu, e_{r}\right\rangle^{2}}{r^{2}}\right\} \\
& =m\left\{2(p+n) \frac{\left\langle\nu, e_{r}\right\rangle^{2}}{r^{2}}+\frac{(p-2)(p+n)}{r^{2}}\right\} \geq 0
\end{aligned}
$$

since $p \geq 2$. This proves the lemma.

We can also prove, that a weaker form of starshapedness is preserved. 
Definition 3.27. A curve $\gamma \in \mathbb{C}^{*}$ will be called austere, if

$$
\left\langle\nu, e_{r}\right\rangle>-1
$$

for all points on $\gamma$.

This means that the angle $\beta$ defined by $\cos \beta=\left\langle\nu, e_{r}\right\rangle$ is contained in the open interval $(-\pi, \pi)$.

Lemma 3.28. If the initial curve $\gamma_{0} \subset \mathbb{C}^{*}$ is austere, then this holds true as long as a smooth solution of (困) exists in $\mathbb{C}^{*}$.

Proof. From equations (3.16), (3.18) and the evolution equation of $\left\langle\nu, e_{r}\right\rangle$ one easily deduces the evolution equation of $\beta$, i.e.

$$
\frac{d}{d t} \beta=\Delta \beta+\frac{n-1}{r}\left\langle\nabla \beta, e_{r}\right\rangle+\frac{n}{r^{2}} \sin (2 \beta) .
$$

Then, using the periodicity of $\sin (2 \beta)$ and the estimate $\sin x \leq \frac{2 x}{\pi}$, for $-\frac{\pi}{2} \leq x \leq 0$ we conclude

$$
\frac{d}{d t}(\beta-\pi) \leq \Delta(\beta-\pi)+\frac{n-1}{r}\left\langle\nabla(\beta-\pi), e_{r}\right\rangle+\frac{4 n}{\pi r^{2}}(\beta-\pi)
$$

for all $-\frac{\pi}{4} \leq \beta-\pi \leq 0$. The parabolic maximum principle implies that $\beta-\pi$ stays negative as long as $\frac{n}{r^{2}}$ stays bounded. The estimate $\beta>-\pi$ can be derived similarly.

In view of Lemma 3.28 we also add the following lemma.

Lemma 3.29. If $z: S^{1} \rightarrow \mathbb{C}^{*}$ is a closed, austere and tamed curve, then $z$ is starshaped and the function

$$
\delta:=r^{n}\left\langle\nu, e_{r}\right\rangle
$$

satisfies

$$
\delta \geq \rho^{n}
$$

where

$$
\rho:=\min _{\phi \in S^{1}} r(\phi)
$$

Proof. By (3.18) we have

$$
d \delta=r^{n} f d r
$$

and since $f>0$ we must have $|\nabla r|^{2}=1-\left\langle\nu, e_{r}\right\rangle^{2}=0$ at a minimum of $\delta$. Since $z$ is austere, we conclude that at such a point $\left\langle\nu, e_{r}\right\rangle=1$ and that the minimum of $\delta$ is bounded from below by $\rho^{n}>0$. Hence $z$ must also be starshaped. 


\section{Singularities For StARShaped PROFile CURVES}

In this section we will prove Theorems 1.14 and 1.15

Proof of Theorem 1.14. First we recall that $r>0$ on $\left[0, T_{\text {sing }}\right.$ ) since $n \geq 2$ (see Lemma 3.1). We will prove that $\sup |f|<\infty$ as long as inf $r>0$. In view of Lemma 3.1 this will prove the theorem. Let $\beta$ be the angle determined by $\cos \beta=\left\langle\nu, e_{r}\right\rangle$ and suppose $p=p(\beta)$ is an arbitrary smooth function of $\beta$ which will be determined later. We will denote a partial derivative w.r.t. $\beta$ by a prime. The evolution equation for $s:=f p$ is given by

$$
\begin{aligned}
\frac{d}{d t} s= & \Delta s+\frac{n-1}{r}\left\langle\nabla s, e_{r}\right\rangle-2\langle\nabla p, \nabla f\rangle \\
& +s\left(k^{2}-\frac{p^{\prime \prime}}{p}\left(k-\frac{\left\langle\nu, e_{r}\right\rangle}{r}\right)^{2}\right. \\
& \left.+\frac{n-1}{r^{2}}\left(2\left\langle\nu, e_{r}\right\rangle^{2}-1\right)+\frac{n p^{\prime}}{r^{2} p} \sin (2 \beta)\right) \\
= & \Delta s+\frac{n-1}{r}\left\langle\nabla s, e_{r}\right\rangle-\frac{2}{p}\langle\nabla p, \nabla s\rangle \\
& +s\left(k^{2}+\left(2\left(\frac{p^{\prime}}{p}\right)^{2}-\frac{p^{\prime \prime}}{p}\right)\left(k-\frac{\left\langle\nu, e_{r}\right\rangle}{r}\right)^{2}\right. \\
& \left.+\frac{n-1}{r^{2}}\left(2\left\langle\nu, e_{r}\right\rangle^{2}-1\right)+\frac{n p^{\prime}}{r^{2} p} \sin (2 \beta)\right) .
\end{aligned}
$$

Suppose $\liminf \operatorname{in}_{t \rightarrow T_{\text {sing }}}\left(\min _{\gamma_{t}} r\right)>0$. Then by Lemma 3.26 we can find $\sigma>1, \epsilon>0$ such that $\cos (\sigma \beta) \geq \epsilon>0$ holds on $\left[0, T_{\text {sing }}\right)$. Now we choose $p=\frac{1}{\cos (\sigma \beta)}$. Then $2\left(\frac{p^{\prime}}{p}\right)^{2}-\frac{p^{\prime \prime}}{p}=-\sigma^{2}$ gives

$$
\begin{aligned}
\frac{d}{d t} s= & \Delta s+\frac{n-1}{r}\left\langle\nabla s, e_{r}\right\rangle-\frac{2}{p}\langle\nabla p, \nabla s\rangle \\
& +s\left(k^{2}-\sigma^{2}\left(k-\frac{\left\langle\nu, e_{r}\right\rangle}{r}\right)^{2}\right. \\
& \left.+\frac{n-1}{r^{2}}\left(2\left\langle\nu, e_{r}\right\rangle^{2}-1\right)+\frac{n \sigma}{r^{2}} \tan (\sigma \beta) \sin (2 \beta)\right) .
\end{aligned}
$$

Again, since $r$ is bounded from below and since $\sigma>1$, there exists a small positive constant $\rho$ and a positive constant $c$ depending on $n, \sigma, \epsilon, \inf r \operatorname{such}$ that

$$
\begin{aligned}
& k^{2}-\sigma^{2}\left(k-\frac{\left\langle\nu, e_{r}\right\rangle}{r}\right)^{2} \\
& +\frac{n-1}{r^{2}}\left(2\left\langle\nu, e_{r}\right\rangle^{2}-1\right)+\frac{n \sigma}{r^{2}} \tan (\sigma \beta) \sin (2 \beta) \\
& \leq-\rho s^{2}+c .
\end{aligned}
$$


Substituting this in the evolution equation for $s$, we may apply the maximum principle on $\left[0, T_{\text {sing }}\right)$ to see that $s$ must be bounded from above and from below on the time interval $\left[0, T_{\text {sing }}\right)$. This implies that $|f|$ and $|k|$ are uniformly bounded on $\left[0, T_{\text {sing }}\right)$ as well which contradicts $T_{\text {sing }}<\infty$.

Now let us assume that $\gamma \in \mathbb{C}^{*}$ is a smooth, starshapedly immersed curve with winding number $\omega_{0}:=$ wind $_{0}(z) \cdot \gamma$ can be parameterized by a smooth map

$$
\begin{aligned}
z & : \quad S^{1} \rightarrow \mathbb{C} \\
z(\phi) & :=r(\phi)\left(\begin{array}{l}
\cos \left(\omega_{0} \phi\right) \\
\sin \left(\omega_{0} \phi\right)
\end{array}\right),
\end{aligned}
$$

where $r$ is a positive smooth function on $S^{1} \cong[0,2 \pi)$. We abbreviate a derivative w.r.t. $\phi$ by a prime. Then

$$
\begin{gathered}
z^{\prime}(\phi)=r^{\prime}(\phi)\left(\begin{array}{c}
\cos \left(\omega_{0} \phi\right) \\
\sin \left(\omega_{0} \phi\right)
\end{array}\right)+\omega_{0} r(\phi)\left(\begin{array}{c}
-\sin \left(\omega_{0} \phi\right) \\
\cos \left(\omega_{0} \phi\right)
\end{array}\right), \\
\nu(\phi)=\frac{1}{\sqrt{g(\phi)}}\left(\omega_{0} r(\phi)\left(\begin{array}{c}
\cos \left(\omega_{0} \phi\right) \\
\sin \left(\omega_{0} \phi\right)
\end{array}\right)-r^{\prime}(\phi)\left(\begin{array}{c}
-\sin \left(\omega_{0} \phi\right) \\
\cos \left(\omega_{0} \phi\right)
\end{array}\right)\right),
\end{gathered}
$$

where $\nu$ is the outward pointing unit normal and $g$ denotes the induced metric on the curve given by

$$
g(\phi)=\left\langle z^{\prime}(\phi), z^{\prime}(\phi)\right\rangle=\left(\omega_{0} r(\phi)\right)^{2}+\left(r^{\prime}(\phi)\right)^{2} .
$$

The formula for the curvature $k$ is

$$
k=\frac{1}{g}\left\langle z^{\prime \prime}, \nu\right\rangle=\frac{\omega_{0}}{\sqrt{g}}\left(1+\frac{\left(r^{\prime}\right)^{2}-r r^{\prime \prime}}{g}\right) .
$$

Moreover,

$$
\frac{\langle z, \nu\rangle}{|z|^{2}}=\frac{\omega_{0}}{\sqrt{g}}
$$

and

$$
f=k+(n-1) \frac{\langle z, \nu\rangle}{|z|^{2}}=\frac{\omega_{0}}{\sqrt{g}}\left(n+\frac{\left(r^{\prime}\right)^{2}-r r^{\prime \prime}}{g}\right) .
$$

Let us define the angle $\beta$ by

$$
\beta:=\frac{1}{\omega_{0}} \arctan \frac{r^{\prime}}{\omega_{0} r} .
$$

We obtain

$$
\beta^{\prime}=\frac{r r^{\prime \prime}-\left(r^{\prime}\right)^{2}}{\left(\omega_{0} r\right)^{2}+\left(r^{\prime}\right)^{2}}
$$

so that with (4.6) we get

$$
f \sqrt{g}=\omega_{0}\left(n-\beta^{\prime}\right) .
$$

$\beta$ measures how much the curves differ from a circle centered at the origin. If $\gamma$ is tamed, then $f>0$. This is equivalent to $(n \phi-\beta)^{\prime}>0$. 
Proof of Theorem 1.15 .

(i) The stability of $\mathscr{E}$ follows directly from Proposition 1.4, Lemma 3.6 Corollaries 3.10, 3.13 and 3.24. The existence of type-1 singularities within $\mathscr{E}$ will be shown below and the existence of type-2 singularities follows from Neves' work [14] in the following way: As pointed out in 14, a straight line in $\mathbb{C}^{*}$ will develop a type-2 singularity in case $n \geq 2$ (cf. Figure 2). Thus, by some glueing technique, we ob-

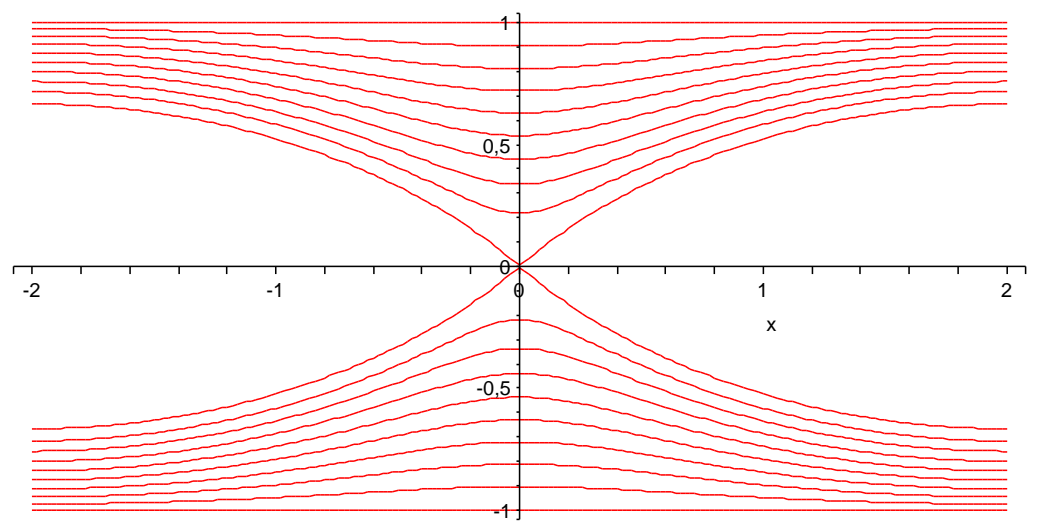

Figure 2. Straight lines in $\mathbb{C}^{*}$ develop a type-2 singularityin case $n \geq 2$.

tain also examples of closed profile curves, that generate Lagrangian submanifolds in $\mathscr{E}$ and which will develop a type-2 singularity at the origin as $t \rightarrow T_{\text {sing }}$ and for which $T_{\text {sing }}<\frac{1}{\varepsilon}$ so that the enclosed area

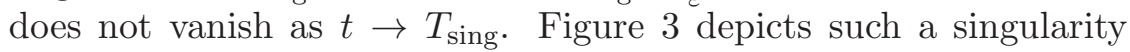
formation.

(ii) Let $\gamma$ be an arbitrary profile curve such that the corresponding Lagrangian submanifold $L$ belongs to $\mathscr{E}$ and such that $\gamma$ is symmetric by rotations of $2 \pi / l$ with $l \geq 1+4 n \omega_{0}$. Let $\phi_{0} \in[0,2 \pi)$ be a point with $r^{\prime}\left(\phi_{0}\right)=0$. W.l.o.g. we may assume $\phi_{0}=0$. Then by symmetry we must have $r^{\prime}(2 \pi / l)=0$ as well. Since $f>0$ the function $n \phi-\beta$ is strictly increasing and from $\beta(0)=\beta(2 \pi / l)=0$ we conclude

$$
0 \leq n \phi-\beta(\phi) \leq \frac{2 n \pi}{l}
$$

for all $\phi \in[0,2 \pi / l)$. Hence $\beta(\phi) \leq \frac{2 n \pi}{l}<\frac{\pi}{2 \omega_{0}}-\frac{\pi}{2 \omega_{0}\left(1+4 n \omega_{0}\right)}$ and $\beta(\phi) \geq n \phi-\frac{2 n \pi}{l}>\frac{\pi}{2 \omega_{0}\left(1+4 n \omega_{0}\right)}-\frac{\pi}{2 \omega_{0}}$. This implies that there exists 


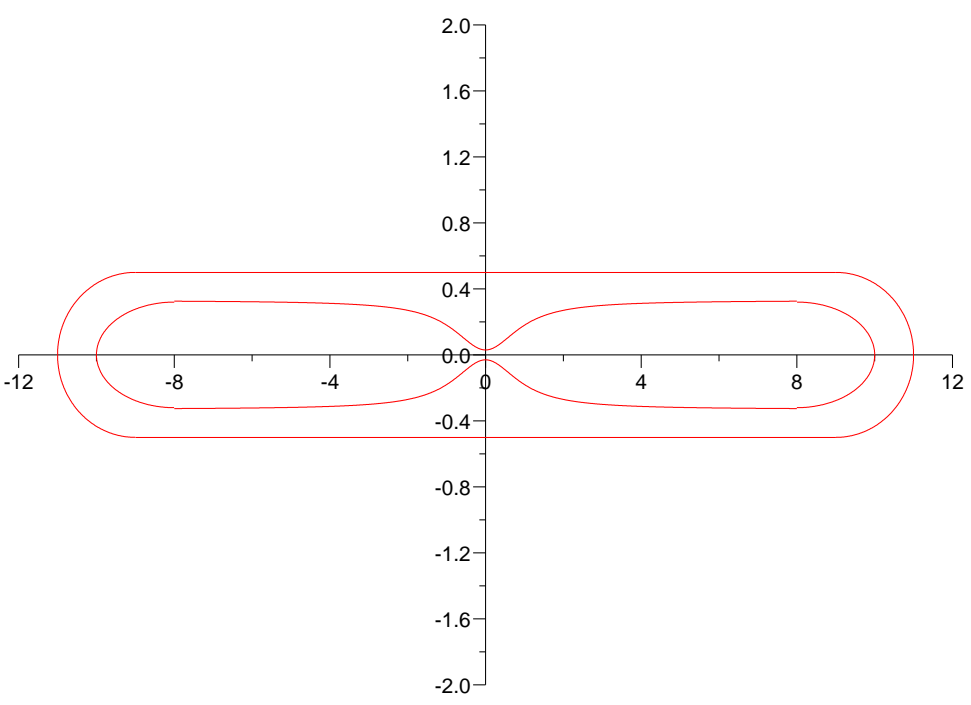

Figure 3. The outer curve will evolve into a curve that roughly looks like the inner one. A type-2 singularity forms.

a constant $c>0$ independent of $\gamma$ such that

$$
c-\frac{\pi}{2} \leq \arctan \frac{r^{\prime}}{\omega_{0} r} \leq \frac{\pi}{2}-c
$$

and therefore

$$
\sup _{\gamma}\left|\frac{r^{\prime}}{r}\right| \leq C
$$

for some uniform constant $C$ independent of $\gamma$. Let $r_{+}(\gamma):=\max _{\gamma} r$, $r_{-}(\gamma):=\min _{\gamma} r$. Integrating (4.10) on $\gamma$ we obtain the Harnack estimate

$$
\frac{r_{+}(\gamma)}{r_{-}(\gamma)} \leq e^{2 \pi C}
$$

which is independent of $\gamma$.

Now for some $\gamma_{0}$ as above let $\gamma_{t}$ be the profile curves given by the equivariant mean curvature flow (函). Since the mean curvature flow preserves the invariance under isometries we conclude from the first part of the proof that $\gamma_{t}$ stays in our class and consequently (4.11) holds for all $t \in\left[0, T_{\text {sing }}\right)$. 
From Theorem 1.14 we get $\liminf _{t \rightarrow T_{\text {sing }}} r_{-}(t)=0$ and 4.11) then implies

$$
\limsup _{t \rightarrow T_{\text {sing }}} r_{+}(t)=0 \text {. }
$$

In particular, $\lim _{t \rightarrow T_{\text {sing }}} A\left(\gamma_{t}\right)=0$ which in view of the symplectic area formula and the monotonicity of $\gamma_{0}$ is only possible, if $T_{\operatorname{sing}}=\frac{1}{\varepsilon}$.

By Proposition 1.8 the rescaled flow

$$
\tilde{z}=\frac{z}{\sqrt{2\left(T_{\text {sing }}-t\right)}}
$$

becomes Hamiltonian and the symplectic area

$$
\tilde{A}(\tilde{z})=\frac{1}{2} \int_{S^{1}}\langle\tilde{z}, \tilde{\nu}\rangle d \tilde{\mu}
$$

is fixed which is equivalent to

$$
\frac{\omega_{0}}{2} \int_{0}^{2 \pi} \tilde{r}^{2}
$$

being fixed. By the mean value theorem and the logarithmic Harnack inequality (4.11) for $r$ and $\tilde{r}$ we then conclude that there exist uniform positive upper and lower bounds for $\tilde{r}$. Similar as in the proof of Theorem 1.14 we can then derive a uniform upper bound for $|\tilde{f}|$. From the definition of the rescaling it follows that $|\tilde{f}|$ is uniformly bounded, if and only if $|A|^{2} \leq \frac{c}{T_{\text {sing }}-t}$ for some constant $c$, so that the singularity must be of type-1. From Huisken's result for the blow-up limits of type-1 singularities we then obtain a smooth closed limiting curve $\tilde{\gamma}_{\infty} \subset \mathbb{C}^{*}$ that is one of the self-similarly contracting solutions classified by Anciaux.

\section{REFERENCES}

1. H. AnCIAux, Construction of Lagrangian self-similar solutions to the mean curvature flow in $\mathbb{C}^{n}$, Preprint (2004).

2. M. Audin, F. Lalonde And L. Polterovich, Symplectic rigidity: Lagrangian submanifolds, in Holomorphic curves in symplectic geometry, vol. 117 of Progr. Math., Birkhäuser, Basel, 1994, pp. 271-321.

3. Y. CheKanov, Lagrangian tori in a symplectic vector space and global symplectomorphisms, Math. Z., 223 (1996), pp. 547-559.

4. J. Chen And J. Li, Singularity of Mean Curvature Flow of Lagrangian Submanifolds, E-print, math.DG/0301281 (2003), 26 pages.

5. K. Cieliebak And E. Goldstein, A note on mean curvature, Maslov class and symplectic area of Lagrangian immersions, Preprint (2004), 5 pages. 
6. Y. EliashberG, Topology of 2-knots in $\mathbf{R}^{4}$ and symplectic geometry, in The Floer memorial volume, vol. 133 of Progr. Math., Birkhäuser, Basel, 1995, pp. 335-353.

7. Y. Eliashberg And L. Polterovich, Local Lagrangian 2-knots are trivial, Ann. of Math. (2), 144 (1996), pp. 61-76.

8. — The problem of Lagrangian knots in four-manifolds, in Geometric topology (Athens, GA, 1993), vol. 2 of AMS/IP Stud. Adv. Math., Amer. Math. Soc., Providence, RI, 1997, pp. 313-327.

9. M. E. GAGE, An isoperimetric inequality with applications to curve shortening, Duke Math. J., 50 (1983) 4, pp. 1225-1229.

10. K. Groh, Contraction of equivariant Lagrangian embeddings under the mean curvature flow, preprint (2006), University of Hannover.

11. M. Gromov, Pseudoholomorphic curves in symplectic manifolds, Invent. Math., 82 (1985), pp. 307-347.

12. G. Huisken, Asymptotic behavior for singularities of the mean curvature flow, J. Differential Geom., 31 (1990) 1, pp. 285-299.

13. J.-M. Morvan, Classe de Maslov d'une immersion lagrangienne et minimalité, C. R. Acad. Sci. Paris Sér. I Math., 292 (1981), pp. 633-636.

14. A. Neves, Singularities of Lagrangian mean curvature flow, preprint (2005).

15. Y.-G. Он, Floer cohomology of Lagrangian intersections and pseudo-holomorphic disks I, Comm. Pure Appl. Math., 46 (1993), pp. 949-993.

16. K. Smoczyk, Self-shrinkers of the mean curvature flow in arbitrary codimension, IMRN, 48 (2005), pp. 2983-3004.

17. - Angle theorems for the Lagrangian mean curvature flow, Math. Z., 240 (2002), pp. 849-883.

18. - Symmetric hypersurfaces in Riemannian manifolds contracting to Lie-groups by their mean curvature, Calc. of Var., 4 (1996), pp. 155-170.

19. K. Smoczyk AND M.-T. WANG, Mean curvature flows for Lagrangian submanifolds with convex potentials, J. Differential Geom., 62 (2002), pp. 243-257.

20. C. Viterbo, A new obstruction to embedding Lagrangian tori, Invent. Math., 100 (1990), pp. 301-320.

21. M.-T. WANG, Mean curvature flow of surfaces in Einstein four-manifolds, J. Differential Geom., 57 (2001), pp. 301-338.

22. K. Zenmisch, Isotopies of Lagrangian tori in symplectic vector spaces - Part III. preprint, Leipzig, January 31, 2005.

Universität Leipzig, Institut für Mathematik, Augustusplatz 10-11, 04109 Leipzig, GERMANY

E-mail address: Matthias.Schwarz@mathematik.uni-leipzig.de

E-mail address: Kai.Zehmisch@mathematik.uni-leipzig.de

Universität Hannover, Institut für Differentialgeometrie, Welfengarten 1, 30167 Hannover, Germany

E-mail address: groh@math.uni-hannover.de

E-mail address: smoczyk@math.uni-hannover.de 
This figure "type2.jpg" is available in "jpg" format from: http://arxiv.org/ps/math/0606428v1 
This figure "type2b.jpg" is available in "jpg" format from: http://arxiv.org/ps/math/0606428v1 
This figure "figure8.jpg" is available in "jpg" format from: http://arxiv.org/ps/math/0606428v1 